Design: Fifteen fresh cases of primary breast carcinoma and 8 HER2+ paraffinembedded core biopsy cases were studied. Fresh tissue was divided into five fragment to approximate a bone core biopsy. One fragment (control) was fixed in $10 \%$ neutra buffered formalin (NF). Remaining fragments were also exposed to formic acid (FA) or hydrochloric acid $(\mathrm{HCl})$ decalcification for 1 or 5 hours. All fragments were embedded in one block and stained for an IHC panel. The known HER2+ cases were exposed to either 1 or 5 hours of FA, and HER2 FISH was also performed. Results were interpreted as follows: H-scores for ER, PR, and GATA-3 were assigned from 0-300, HER2, CK7, GCDFP-15, Pax-8, TTF-1, CK20, and mammaglobin were scored from 0-3+, and Ki67 from $0-100 \%$. Mean scores were compared using t-test for paired samples.

Results: Mean scores for ER, PR, HER2, Ki67 and GATA-3 were significantly lower than NF in tissue after either 1 or 5 hours of HCl. Mean scores for GCDFP-15 staining in tissue after 5 hours of $\mathrm{HCl}$ were significantly lower than NF. No significant differences in mean score were seen between NF and 1 hour FA for any IHC staining. After 5 hours of FA, only Ki67 average score was significantly less than NF.

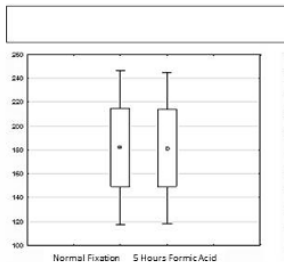

$$
\text { Estrogen Receptor }
$$
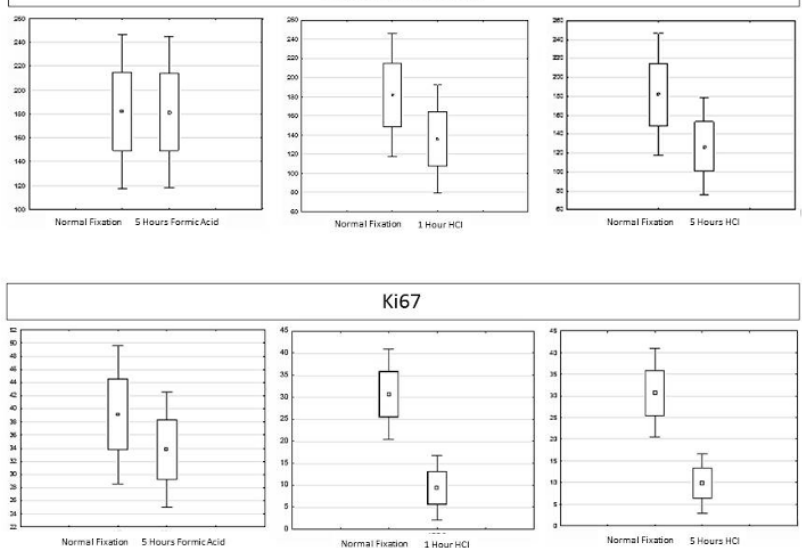

Three of 5 HER 2 equivocal cases ( $2+$ by IHC) were negative $(0$ or $1+)$ following 5 hours of $\mathrm{HCl}$ and $1 / 5$ was negative following FA exposure. All HER2+ cases were amplified by FISH.

Conclusions: Decalcification with $\mathrm{HCl}$ but not FA has significant effects on IHC staining. HER2 FISH was not affected by FA exposure. Breast cancer biomarker interpretation in samples exposed to $\mathrm{HCl}$ should be avoided, but formic acid may be used for 5 hours or less with caution.

\section{Techniques (including Ultrastructure)}

2104 Diagnostic Utility of Fourier Transform Infrared (FT-IR) Spectroscopic Imaging of Preeclamptic Placenta Tissue

Oluwatobi Adelaja, Vishal K Varma, Virgilia Macias, Andre Balla, Michael Walsh. University of Illinois Hospital and Health Sciences Systems, Chicago, IL; University of Illinois at Chicago, Chicago, IL.

Background: The diagnostic criterion for preeclampsia is hypertension (blood pressure $\geq 140 / 90 \mathrm{mmHg}$ ) and proteinuria ( $>300 \mathrm{mg} / 24$ hours) after 20 weeks gestation. Various etiologies including fetal growth restriction, diabetes mellitus and antiphospholipid syndrome share similar histological features of maternal malperfusion. This has made morphological analysis of the placenta via standard optical microscopy limited in diagnostic potential. A recent study demonstrated a 2 -fold yield of histological findings compatible with maternal malperfusion with increased sampling of the preeclamptic (P) placenta. However, increase sampling methodology and nonspecific histological features still leaves a robust biochemical signature unanalyzed. The aim of this study is to assess the biomolecular signature of preeclampsia tissue and propose a novel methodology of evaluating placenta tissue for diagnostic application.

Design: We utilized a placenta tissue microarray (TMA) that were constructed based on the functional anatomy of the placenta into individual cores of the chorionic plate $(\mathrm{P} 1)$, middle chorionic villi (P2), and basal plate (P3) for both preeclamptic $(\mathrm{n}=23)$ and nonpreeclamptic (NP) tissue $(n=26)$. Multivariate techniques such as principal component analysis and linear discrimination analysis were utilized to differentiate the two groups.

Results: Analysis of P1, P2 and P3 were performed independently of one another. We collected FT-IR data from the TMA and extracted biochemical signatures from the structures of the placenta. P1 showed the best biochemical distinction between normal $(n=24)$ and preeclamptic $(n=20)$ placenta, with a misclassification rate of 3 out of 44 samples. P3 also showed good biochemical distinction between $\mathrm{P}(\mathrm{n}=13)$ and NP $(n=21)$ with a misclassification rate of 4 out of 34 samples. Lastly, $\mathrm{P} 2$ showed the least discrimination compared to the other regions of the placenta. $\mathrm{P}(\mathrm{n}=19)$ and $\mathrm{NP}(\mathrm{n}=17)$ groups showed misclassification rate of 5 out of 36 samples.

Conclusions: FT-IR spectroscopy imaging of placenta tissue with statistical pattern recognition of spectra is a novel methodology. Our data suggest that spectra changes can be reliably distinguished when discriminating preeclamptic versus nonpreeclamptic placenta. Although we do not predict that this method will be necessary for routine pathology practice, it may be a useful research discovery tool once the spectral signature is decoded to identify specific molecular markers. Cervical Dysplasia

Lubna A Alattia, Moiz Vora, Junaid Ansari, Menchu Ong, James Cotelingam, Domenico Coppola, Rodney Shackelford. LSU Health Shreveport, Shreveport, LA; Feist-Weiller Cancer Center, Shreveport, LA; Moffitt Medical Group, Tampa, FL

Background: Invasive cervical squamous cell carcinoma (SCC) is the second most common gynecologic malignancy world-wide. Histologically it is almost invariably preceded by progressive grades of in situ dysplasia. Nicotinamide phosphoribosyl transferase (Nampt) catalyses the rate-limiting step of nicotinamide adenine dinucleotide synthesis. Nampt protein levels are elevated in number of epithelial and mesenchymal malignancies in which higher levels of NAMPT expression correlate with advanced tumor grade and worse prognosis. Here we report Nampt expression in a range of cervical epithelial lesions.

Design: We used Tissue microarrays (TMAs) contained 12 benign cervical squamous epithelial samples, 15,15 , and 13 samples of cervical intraepithelial neoplasia (CIN) I, II, III respectively. Tissue cores also included cases of SCC, 5 low grade, 67 intermediate, and 81 high grade. 7 cases of endocervical adenocarcinoma. Additionally, 13 sections of benign endocervical epithelium were also identified within the TMA tissue and used in this analysis. The concentration of primary Nampt antibody was optimized to normal kidney as control tissue. Mouse monoclonal antibody to human Nampt was used at a 1:1000 concentration. Relative Nampt protein expression was determined as immunostain intensity scored on a 0-3 scale. The final IHC score was the product of the percentage of cells stained multiplied by the intensity score, allowing for a maximal score of 9 and a minimal score of 0 .

Results: Nampt is a marker for cervical dysplasia which increases in a grade-dependent manner in CIN and invasive SCC. Nampt is also highly expressed in cervical adenocarcinoma in comparison to benign endocervical epithelium (Table 1). In contras to p16 which is expressed in only $54 \%$ of CIN I, immunoreactivity for Nampt is found to be doubled in CIN I when compared to benign sequamous epithelium.

\begin{tabular}{|l|l|l|l|}
\hline Tissue Type & $\begin{array}{l}\text { Sample } \\
\text { Number }\end{array}$ & $\begin{array}{l}\text { Average Nampt IHC } \\
\text { Score }\end{array}$ & SEM \\
\hline $\begin{array}{l}\text { Benign Cervical Sequamous } \\
\text { Epithelium }\end{array}$ & 14 & 1.57 & 0.14 \\
\hline CIN I & 11 & 3.45 & 0.49 \\
\hline CIN II & 9 & 3.86 & 1.02 \\
\hline CIN III & 8 & 4.91 & 0.76 \\
\hline SCC Grade I & 5 & 4.00 & 0.55 \\
\hline SCC Grade II & 56 & 4.91 & 0.32 \\
\hline SCC Grade III & 53 & 6.43 & 0.37 \\
\hline Benign Cervical Columnar Epithelium & 13 & 1.77 & 0.23 \\
\hline Adenocarcinoma & 7 & 7.71 & 0.95 \\
\hline
\end{tabular}

Conclusions: Nampt is a reliable marker of progression in cervical dysplasia that may contribute towards evaluation of cervical epithelial abnormalities.

2106 Application of Hematoxylin and Eosin Staining as Counter Stain to Improve Immunohistochemistry Interpretation

Roula Albadine, Kevin Loayza-Vega, Eloise Adam-Granger, Andrée-Anne Grosset, Maria-Claudia Vladoiu, Dominique Trudel. CHUM, Montreal, QC, Canada.

Background: Common progressive hematoxylins like Mayer's or common regressive hematoxylins like Harris are used as counterstain for IHC, despite the fact that hematoxylin/eosin (H\&E) combination is the most common staining technique in histology. The aim of the current study is to investigate the clinical utility of $\mathrm{H} \& \mathrm{E}$ as counter staining for IHC (HE-IHC) to facilitate the interpretation of a marker on small biopsies.

Design: The HE-IHC protocol was optimized to ensure quality as well as technical efficiency. With the optimized protocol, we stained multiple prostate and liver cases with class $1 \mathrm{IHC}$ markers (Ck5/6, 34BE12, p63, p504S, p53, Ck7). We also stained tissue microarrays from the Canadian Immunohistochemistry Quality Control (cIQc) proficiency testing exercises for ER, PR, Her2 (Run 59), and p53 (Run 54). Scoring was done by 2 independent pathologists in blind experiment settings. Our staining was considered optimal if results were completely concordant with reference.

Results: The biggest strength of HE-IHC is its ability to identify morphology as in routine HE. Class 1 markers, including dual stains (red/brown), were easily interpretable (Fig 1). Our staining was considered optimal for ER, PR and Her2. There were no false results for Her2. Even cores with weaker staining for ER were stained and interpreted properly. Our staining was considered optimal for $41 / 42$ cores stained with p53 (Fig 2 ); The missed core was interpreted correctly by only $2 / 50$ laboratories participating in the cIQc. 


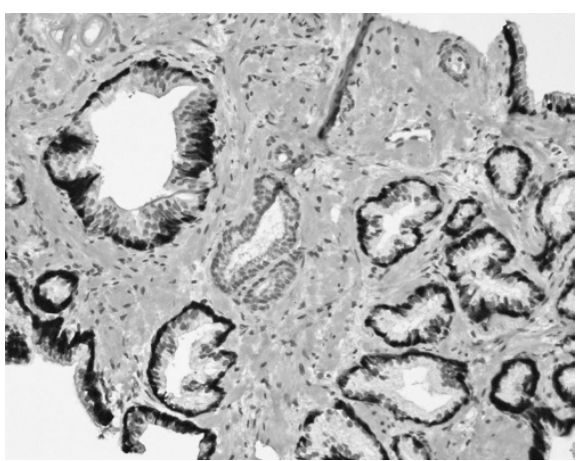

Fig 1. p63/34BE12/p504s in a prostate biopsy.

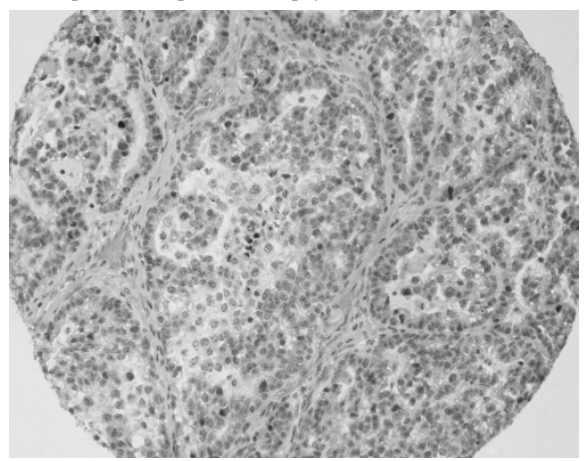

Fig 2. Wild-type p53 in the cIQc TMA.

Conclusions: The H\&E counterstaing enhances IHC interpretation. For precise scoring, grading of cancer, routine application of HE with single or dual color immunostaining is recommended, especially for the evaluation of small biopsies. For class 2 markers, in the view of promising results, our slides will be evaluated by cIQc for quality assurance/ external proficiency testing.

2107 Multiplex Cocktails for Immunotherapy Targets: PD-L1 with Tumor Specific Transcription Factors

David Altree-Tacha, Wei Yuan, George Yang. Biocare Medical, Concord, CA.

Background: Therapeutics targeting of PD-L1 in several cancers are currently in clinical trials, and the U.S. FDA has granted accelerated approval for Keytruda (pembrolizumab) to treat patients with advanced (metastatic) non-small cell lung cancer. Pathology scoring and interpretation methods have been put forth to assay PD-L1 expression, and variability of non-PD-L1 staining across tumors may have important prognostic aspects. PD-L1 expression on tumor cells can be upregulated via activation of CD8+ cytotoxic T lymphocytes. PD-L1 expression is also associated with certain subtypes of tumorassociated lymphocytes, macrophages and dendritic cells. In certain cancers, the cross reactivity of PD-L1 can make interpretation and scoring of these targets difficult. Thus, a strategy using IHC multiplex stains could help resolve challenging cases by cocktailing PD-L1 with transcription factor antibodies along with other strategic markers.

Design: Formalin-fixed paraffin-embedded tissues and tissue microarrays for various targeted tumor types including lung, bladder and melanoma were processed and cut at 4-5 microns. PD-L1 rabbit monoclonal antibody were cocktailed with the following antibodies: TTF-1; CD163; p40 + GATA3; and SOX10. Cocktails were detected with double stain detection system on an automated platform using brown, red and blue chromogens for visualization. Sections were counterstained with a standard hematoxylin or Wiegert's iron hematoxylin.

Results: PD-L1 and TTF-1 and PD-L1 + CD163 cocktails were successfully performed on various lung cancers. The nuclear staining of TTF-1 in lung adenocarcinoma (blue chromogen) helped define tumor positive PD-L1 (DAB) positive cells (Figure 1), and CD163 (Fast Red) marked PD-L1 positive macrophages. Co-expression or urothelial makers p $40+$ GATA3 identified tumor positive PD-L1 in bladder cancers. Finally, SOX10 nuclear staining was observed in most melanoma cells and could be easily separated with orwithout PD-L1 membrane staining.

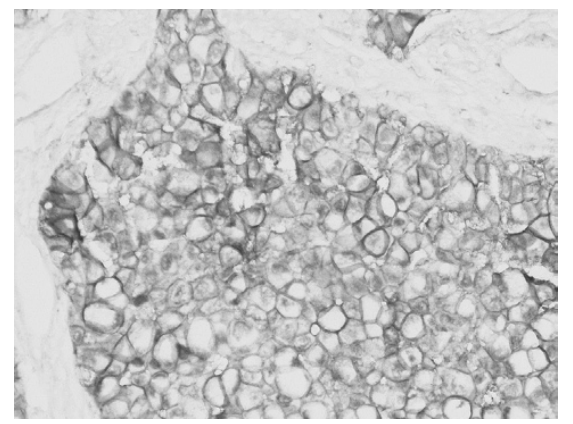

Figure 1: Lung adenocarcinoma stained with TTF-1 (blue) and PD-L1 (brown)
Conclusions: PD-L1 cocktailed with strategic nuclear or cytoplasmic antibodies can help discriminate tumor cells from non-tumor cells, and may facilitate quantitation or scoring methods for more accurate assessment of this key immunotherapy marker.

\section{Role of pHLIP Imaging in Urological Malignancies}

Ali Amin, Jovana Golijanin, Anna Moshnicova, Joseph Brito, Timothy Tran, RamonaCosmina Adochite, Oleg A Andreev, Troy Crawford, Donald M Engelman, Gregory Andreev, Yana K Reshetnyak, Dragan Golijanin. Brown University, Providence, RI; University of Rhode Island, North Kingston, RI; Yale University, New Haven, CT.

Background: Accurate \& timely detection of malignancy is crucial for successful management \& better outcome. Most efforts are focused on identification of highly sensitive and specific, tumor-specific markers. But cancer cells show phenotype change with expression of unpredictable new molecular alterations.

Acidity due to high proliferation, turnover and metabolism is a universal feature of cancer cells. $p H$ Low Insertion Peptide ( $p H L I P$ ) detects elevated intracellular acidity and can label malignant cells using a non-tumor-specific mechanism.

Design: 25 urologic tumors were selected in The Miriam Hospital, RI. Separate consents for radical surgery and research protocol were obtained. The organs were irrigated $e x$ vivo with saline (using catheter in bladder and arterial infusion in kidney), followed by 60 minute incubation with ICG-pHLIP conjugate. After irrigation, the specimen was viewed using Near Infra-red scope on daVinci Robot. Any labeled area was marked and processed for histopathology individually.

Results: 29 lesions in 22 cystectomies and 3 lesions in 3 nephrectomies were evaluated. Age: 51-84 (mean 67.7) years, $\mathrm{M} / \mathrm{F}=20 / 5$. Table 1 contains demographics, diagnosis, stage and imaging results.

\begin{tabular}{|c|c|c|c|c|c|c|}
\hline $\begin{array}{c}\text { Case } \\
\#\end{array}$ & $\begin{array}{c}\text { Sex/age } \\
\text { (y) }\end{array}$ & $\begin{array}{l}\text { Pathological } \\
\text { stage }\end{array}$ & Pathological diagnosis & $\begin{array}{l}\text { Lasion } \\
\quad \|\end{array}$ & $\begin{array}{l}\text { White light } \\
\text { diagnosis }\end{array}$ & $\begin{array}{c}\text { Fluorescence } \\
\text { inaging }\end{array}$ \\
\hline 1 & $M / 63$ & pr3an1 & $\begin{array}{l}\text { Infiltrating high-grade } \\
\text { urothelial carcinoma, crs }\end{array}$ & 1 & + & + \\
\hline 2 & $\mathrm{M} / 61$ & PTONO & $\begin{array}{l}\text { Diverticulum with urothelial } \\
\text { atypia }\end{array}$ & & + & + \\
\hline \multirow[t]{2}{*}{3} & \multirow[t]{2}{*}{$F / 84$} & \multirow[t]{2}{*}{ YPT3bNO } & $\begin{array}{l}\text { Invasive high-grado } \\
\text { urothelial careinoma }\end{array}$ & 2 & + & + \\
\hline & & & $\begin{array}{l}\text { Invasive high-grade } \\
\text { urothelial carcinoma }\end{array}$ & 3 & + & + \\
\hline 4 & $M / 51$ & pт2aN1 & $\begin{array}{l}\text { Infiltrative high-grade } \\
\text { urothelial carcinoma } \\
\text { micropapillary features }\end{array}$ & 4 & + & + \\
\hline 5 & $\mathrm{M} / 69$ & pTano & $\begin{array}{l}\text { Moninvasive high-grade } \\
\text { papillary careinoma }\end{array}$ & 5 & + & + \\
\hline \multirow[t]{3}{*}{6} & \multirow[t]{3}{*}{$\mathrm{M} / 65$} & \multirow[t]{3}{*}{ pTINo } & $\begin{array}{l}\text { Invasive high-grado } \\
\text { urothelial carcinoma, cIs }\end{array}$ & 6 & + & + \\
\hline & & & crs & 7 & + & + \\
\hline & & & crs & 8 & + & + \\
\hline 7 & $M / 61$ & pTINO & $\begin{array}{l}\text { Invasive high-grade } \\
\text { urothelial caroinoma }\end{array}$ & 9 & + & + \\
\hline \multirow[t]{2}{*}{8} & \multirow[t]{2}{*}{$M / 79$} & \multirow[t]{2}{*}{ prino } & Dysplasia & 10 & - & + \\
\hline & & & $\operatorname{ccc} G$ & & - & + \\
\hline 9 & $\mathrm{M} / 74$ & pTONO & $\operatorname{ccc} \theta$ & & - & + \\
\hline \multirow[t]{4}{*}{10} & \multirow[t]{4}{*}{$F / 82$} & \multirow[t]{4}{*}{ pTiNo } & $\begin{array}{l}\text { Noninvasive high-grade } \\
\text { urothelial caroinoma }\end{array}$ & 11 & + & + \\
\hline & & & $\begin{array}{l}\text { Invasive high-grado } \\
\text { urothelial carcinoma }\end{array}$ & 12 & + & - \\
\hline & & & cIs & 13 & - & + \\
\hline & & & CIS and CCCG & 14 & - & + \\
\hline \multirow[t]{2}{*}{11} & \multirow[t]{2}{*}{$M / 68$} & \multirow[t]{2}{*}{ prisso } & $\mathrm{CIS}$ and $\mathrm{CCCG}$ & 15 & + & + \\
\hline & & & crs & 16 & - & + \\
\hline \multirow[t]{4}{*}{12} & \multirow[t]{4}{*}{$M / 71$} & \multirow[t]{4}{*}{ pTisNo } & $\begin{array}{l}\text { Noninvasive high-grade } \\
\text { urothelial carcinoma }\end{array}$ & 17 & + & + \\
\hline & & & $\begin{array}{l}\text { Noninvasive high-grade } \\
\text { urothelial carcinoma }\end{array}$ & 18 & + & + \\
\hline & & & crs & 19 & - & + \\
\hline & & & crs & 20 & - & + \\
\hline \multirow[t]{2}{*}{$13^{\circ}$} & \multirow[t]{2}{*}{$M / 66$} & \multirow[t]{2}{*}{ pT3N1 } & $\begin{array}{l}\text { Invasive high-grade } \\
\text { urothelial carcinoma }\end{array}$ & & + & ICG-Cys \\
\hline & & & Necrosis, CCCG & & + & \\
\hline 14 & $M / 66$ & prino & $\begin{array}{l}\text { Noninvasive high-grade } \\
\text { urothelial careinoma }\end{array}$ & 21 & + & + \\
\hline 15 & $\mathrm{M} / 57$ & pTino & $\begin{array}{l}\text { Invasive high-grade } \\
\text { urothelial careinoma }\end{array}$ & 22 & + & + \\
\hline Case & $\begin{array}{l}\text { Sex/age } \\
\text { (y) }\end{array}$ & $\begin{array}{l}\text { Pathological } \\
\text { stage }\end{array}$ & Pathological diagnosis & $\begin{array}{l}\text { Lesion } \\
\quad \|\end{array}$ & $\begin{array}{l}\text { White light } \\
\text { diagnosis }\end{array}$ & $\begin{array}{c}\text { Fluorescence } \\
\text { imaging }\end{array}$ \\
\hline \multirow[t]{2}{*}{16} & \multirow[t]{2}{*}{$F / 77$} & \multirow[t]{2}{*}{ pTisNo } & CIs with early invasion & 23 & + & + \\
\hline & & & crs with early invasion & 24 & - & + \\
\hline 17 & $M / 57$ & pTIbNO & $\begin{array}{l}\text { Invasive high-grade } \\
\text { urothelial careinoma }\end{array}$ & 25 & + & + \\
\hline & & & CIs with oarly invasion & 26 & + & + \\
\hline & & & Nocrosis & & + & + \\
\hline $18^{\prime}$ & $\mathrm{M} / 72$ & prano & $\begin{array}{l}\text { Invasive high-grade } \\
\text { urothelial caroinoma, crs }\end{array}$ & 27 & + & + \\
\hline & & & Necrosis in diverticulum & & + & - \\
\hline 19 & $M / 64$ & praano & $\begin{array}{l}\text { Invasive high-grade } \\
\text { urothelial careinoma, cIs, }\end{array}$ & & + & ICG-Cys \\
\hline 20 & $M / 63$ & ypTONO & $\operatorname{ccc} G$ & & + & ICG-Cys \\
\hline 21 & $M / 74$ & pr3aso & $\begin{array}{l}\text { Invanive high-grade } \\
\text { urothelial carcinona }\end{array}$ & 28 & + & + \\
\hline & & & Necrosis & & + & + \\
\hline 22' & $M / 66$ & ypt3ano & $\begin{array}{l}\text { Invasive high-grade } \\
\text { urothelial carcinoma with } \\
\text { neuroendocrine features }\end{array}$ & 29 & + & + \\
\hline 23 & $M / 74$ & pT2BNx & Chromophobe RCC & 30 & + & + \\
\hline 24 & $F / 71$ & pt3anx & C10ar coll RCC & 31 & + & + \\
\hline 25 & $F / 67$ & pT1Nx & $\begin{array}{l}\text { Invasive high-grade } \\
\text { urothelial carcinoma }\end{array}$ & 32 & + & + \\
\hline
\end{tabular}


No adverse morphological change was noted after incubation. In bladder, sensitivity \& specificity of $97 \% \& 80 \%$ were detected respectively, with false-positive result in tumor necrosis and cystitis cystica. A urothelial dysplasia case was detected by the peptide. One upper tract urothelial carcinoma and 2 RCC cases showed strong labeling with ICG-pHLIP without any false-positive result.

Conclusions: $\mathrm{pHLIP}$ peptide detects urological malignancies with a high sensitivity and acceptable specificity. This has diagnostic and therapeutic implications since the peptide can be labeled by anti-tumor chemicals for targeted therapy with reduced toxicity.

2109 Detection of Acute Myeloid Leukemia on Flow Cytometry Data from 351 Subjects Using Machine Learning Technology

Cesar Angeletti. Logical Cytometry, Atlanta, GA.

Background: Flow cytometry is a technique widely used for the analysis of cell populations in the study and diagnosis of human diseases. Although routinely used on hematological specimens, it has been increasingly applied to other fields of medicine. Currently, interpretation of flow cytometry data is performed by an experienced operator mainly through visual selection of subpopulations of interest. The approach is timeconsuming and subjective, sometimes involving intuition rather than standardized statistical inference. Depending of the expertise of the operator, a certain cell population can be misidentified, overestimated or underestimated. Here, an innovative approach using machine learning technology for the interpretation of flow cytometry data is described.

Design: Flow cytometry data was obtained from the public website www.flowrepository org. Flow cytometry files of 316 normal subjects and 43 patients with acute myeloid leukemia (AML) were extracted from its repository \#FR-FCM-ZZYA(AML, FlowCAP II) and transformed using R platform statistical software with addition of the "prada" package (www.bioconductor.org/packages/release/bioc/html/prada.html). Flow cytometry data of the first four normal subjects (datasets 1-4) and the first four AML patients (datasets $5,7,9,26$ ) in the repository were used to create a random training set. The remaining of the repository cohort, composed of 312 normal subjects and $39 \mathrm{AML}$ patients, was used as testing set.

Results: Initial data analysis using seven cell distinction parameters showed good discrimination of normal subjects and AML patients with a calculated receiver operator characteristic area under the curve of 0.917 and a combined sensitivity and specificity of up to $84.6 \%$ and $84.9 \%$, respectively.

Conclusions: Future analysis involving alternate learning parameters are also discussed. The present results suggest that machine learning systems hold a great promise in the interpretation of hematological flow cytometry data.

2110 PD-L1 Expression in Male Breast Carcinoma: Comparison of RNA In Situ Hybridization Technology vs Immunohistochemistry

Anand C Annan, Momin T Siddiqui, Sonal Bhimji-Pattni, Cynthia Cohen. Emory University School of Medicine, Atlanta, GA.

Background: Breast cancer in men is rare with very few therapeutic options Programmed death-ligand 1 (PD-L1) is a transmembrane protein whose over-expression in cancer has been associated with increased aggressiveness and poor prognosis. Newer targeted anti PD-L1 therapies have shown promise in cancer clinical trials. In this study, we evaluated PD-L1 expression in male breast carcinomas by using two different techniques, RNA in situ hybridization (ISH) and immunohistochemistry for PD-L1.

Design: A search of the Anatomic Pathology files at our institution between 2011 to 2016 yielded 23 male breast cancer cases (6 invasive ductal carcinoma [IDC], 5 DCIS 8 IDC with DCIS, 1 pleomorphic lobular carcinoma, 1 solid papillary carcinoma, and 2 metastatic carcinoma). RNA ISH using a novel multiplex nucleic acid method (RNAscope $^{\circledR}$, Leica BioSystems, Buffalo Grove, IL), probe Hs-CD274 (Advanced Cell Diagnostics, Newark, CA), and the Bond III autostainer (Leica Biosystems), and immunohistochemistry for PD-L1 (E1L3N ${ }^{\circledR}, 1: 200$ dilution, Cell Signaling Technology, Danvers, MA) were performed with appropriate controls (for RNA ISH: positive control probe - PP1B, negative control probe - DapB; for IHC: PD-L1 positive and negative lung adenocarcinoma). Presence or absence of staining (intensity and percentage positivity) was documented.

Results: Twelve of the twenty-three cases (52\%) had positive PD-L1 expression by RNA in situ hybridization. All the positive cases had a 3+ (strong) staining intensity, and the percentage positivity ranged from $1 \%$ to $20 \%$. Six of six IDC $(100 \%), 2$ of 5 DCIS (40\%), 3 of 8 IDC with DCIS (38\%), and 1 of 1 pleomorphic lobular carcinoma $(100 \%)$ were positive for PD-L1. In contrast, none of these 23 cases had positive PD-L1 expression by immunohistochemistry.

Conclusions: Novel anti-PD-L1 therapy has shown exciting results, with FDA approval being available for the treatment of melanoma, lung adenocarcinoma and other malignancies. Our study on male breast cancer shows that RNAscope ${ }^{\circledR}$ in situ hybridization technique is superior in detecting PD-L1 expression compared to IHC staining using E1L3N ${ }^{\circledR}$. Our study also shows promising PD-L1 expression in IDC in men $(9 / 14$ cases, $64 \%)$ that can be exploited as a possible therapeutic target using anti-PD-L1 therapy.

\section{MicroRNA Profiling Complements Microscopic/Mutational} Analysis by Defining Alternative Pathways of Carcinogenesis

Joseph F Annunziata, Anna Banizs, Christina M Narick, Sara Jackson, Jan F Silverman, Sydney Finkelstein. Allegheny General Hospital, Pittsburgh, PA; Interpace Diagnostics, Pittsburgh, PA.

Background: Thyroid follicular cell neoplasia is biologically heterogeneous ranging from benign to indolent malignancy to high grade cancer. Mutation genotyping is effective at predicting tumor aggressiveness. Aggressive disease can be seen in a significant minority of cases where common driver mutations are undetected. We performed a correlative analysis of microRNA profiling with specific patterns of thyroid follicular cell nodules using a large clinical case database $(n=5,210)$ seeking discriminating information.

Design: Data was retrospectively mined from a large clinical testing cohort of FNA thyroid nodule cytology. Cytology was based on pathologist Bethesda diagnostic classification. Mutation analysis targeted common mutations (BRAF, ras, PIK3CA, PAX8/PPAR and RET/PTC translocations) by next generation (Illumina). RNA expression classifier utilized a 10 miRNA panel consisting of 5 miRs with increased expression in cancer and 5 miRs with hyperexpression in benign states.

Results: Mutation analysis defined six groups for correlative miR profiling: BRAF+ (aggressive), $\mathrm{N} / \mathrm{H} / \mathrm{K}$ ras + (indolent to aggressive), PAX8/PPARgamma translocation+ (indolent to aggressive) and No detectable mutation yet aggressive. BRAF+ compared to ras + groups showed distinct profiling differences for 7 of 10 individual miRs. No detectable mutation/aggressive subset closely matched BRAF+ supporting common molecular pathway involvement (BRAF phenotype). Among the different ras + nodules, NRAS and HRAS showed a nearly matching miR profile for all 10 miRs. KRAS profiling differences were detected for 5 miRs in keeping with potentially differing biology of this form of ras mutation. PAX8/PPARgamma displayed profiling differences for 8 miR distinct from both BRAF and ras groups. Cytology diagnoses were significant for $\mathrm{B} 5$ and $\mathrm{B} 6$ in $\mathrm{BRAF}+$ cases but otherwise molecular patterns were not predicted by microscopic classification.

Conclusions: Oncogene driver mutations can predict biological aggressiveness including benign versus malignant status in a proportion of thyroid follicular nodules that are indeterminate after cytology evaluation. BRAF mutated nodules display a unique miR expression profile distinct from other common mutations. This BRAF profile is present in mutation negative aggressive disease suggesting BRAF mutational heterogeneity across the nodule with sampling variation. An approach combining RNA expression profiling to define distinct molecular pathways and mutational genotype is well suited to complement microscopic assessment of thyroid neoplasia.

2112 Nanostring Copy Number Variation Assay Is Very Sensitive in Identifying EGFR Amplification but Is Less Sensitive in Identifying Deletions in 1p/19q and PTEN Compared to FISH in Brain Tumors Samples Hanan Armanious, Iyare Izevbaye. University of Alberta, Edmonton, AB, Canada. Background: CNV (copy number variation) is frequently detected in neuro-oncologic tumors and provide important diagnostic, prognostic and predictive information. These changes include EGFR amplification, 1p/19q and PTEN deletion. FISH remains the gold standard for $\mathrm{CNV}$ detection in current molecular pathology practice. However, FISH is costly, time consuming, requires significant manpower and multiple sequentia runs because of its limited ability to multiplex different targets. Newer copy number variation techniques such as Nanostring nCounter may provide a superior testing methodology, combining high-throughput testing, multiplex capability, rapid turnaround time and cost effectiveness.

Design: Here we used a new technology, the Nanostring nCounter CNV assay, which detects $\mathrm{CNV}$ by interrogating hundreds of loci across different regions in each chromosome in a single reaction. DNA was extracted from 23 archival formalin fixed paraffin embedded neuro-oncologic specimen with histologic diagnosis by a neuropathologist and confirmed FISH results. The histologic types include oligodendroglioma, glioblastoma and oligoastrocytoma. FISH results include 16 cases for EGFR amplification; 16 cases for 1p/19q co-deletion and 8 cases for PTEN deletion. 3 samples of normal brain tissue were used as controls. Samples were run on the nCounter $\mathrm{CNV}$ assay and analyzed by the nSolver software. Ratio of target to normalized control was used to identify copy number changes. The results were compared with FISH to determine assay sensitivity and specificity.

Results: Comparison of CNV nanostring assay vs FISH showed $100 \%$ concordance for EGFR amplification ( $6 / 6$ positive and $8 / 8$ negative) with sensitivity, specificity and positive predictive values of $100 \% ; 1 \mathrm{p} / 19 \mathrm{q}$ deletion vs FISH showed $(10 / 12$ positive and $3 / 4$ negative) with sensitivity of $83 \%$, specificity of $75 \%$ and positive predictive value of $91 \%$; PTEN showed (4/4 positive and $3 / 4$ negative) with sensitivity of $100 \%$, specificity of $75 \%$ and positive predictive value of $80 \%$.

Conclusions: These results demonstrate the ability of the CNV nanostring assay to detect copy number variations particularly EGFR amplification, with comparable sensitivity to the reference FISH technique in brain tumor samples. The assay also demonstrated rapid turn around time, high throughput and cost effectiveness in assessing copy number changes. However, more assay optimization is required especially to improve deletion detection.

\section{Rapid Loss of RNA Detection by In Situ Hybridization in Stored} Tissue Blocks and Preservation by Cold Storage of Unstained Slides

Javier A Baena Del Valle, Qizhi Zheng, Jessica Hicks, Helen Fedor, Bruce J Trock, Colm Morrissey, Eva Corey, Toby C Cornish, Karen S Sfanos, Angelo M De Marzo. The Johns Hopkins University, Baltimore, MD; Fundacion Santa Fe De Bogota, Bogota DC, Colombia; University of Washington, Seattle, WA; University of Colorado School of Medicine, Aurora, CO.

Background: Novel methods for RNA in situ hybridization (RISH) using dual probes (Z-pairs) and branched DNA amplification are being widely adapated for research and clinical use on FFPE tissues with a great potential for unlocking vast stores of existing biospecimens for localization and quantification of any RNA species. RNA extracted from FFPE tissues decreases in quality with time stored at room temperature. Therefore, it is important to study the effects of tissue block age on this Z-pair/branched DNA in situ hybridization technology.

Design: We examined the effects of tissue block age on FFPE tissues from prostatectomy specimens using a number of RISH probes (TP63, PTEN, MYC, PCA3). We then examined the effects of block age in a tissue microarray (TMA) experiment with 
LuCaP patient derived xenograft tissues (PDXs). Finally, we performed a prospective quantitative TMA-based experiment to test the effects of storage condistion for unstained FFPE slides to determine whether loss of signals could be prevented using cold storage.

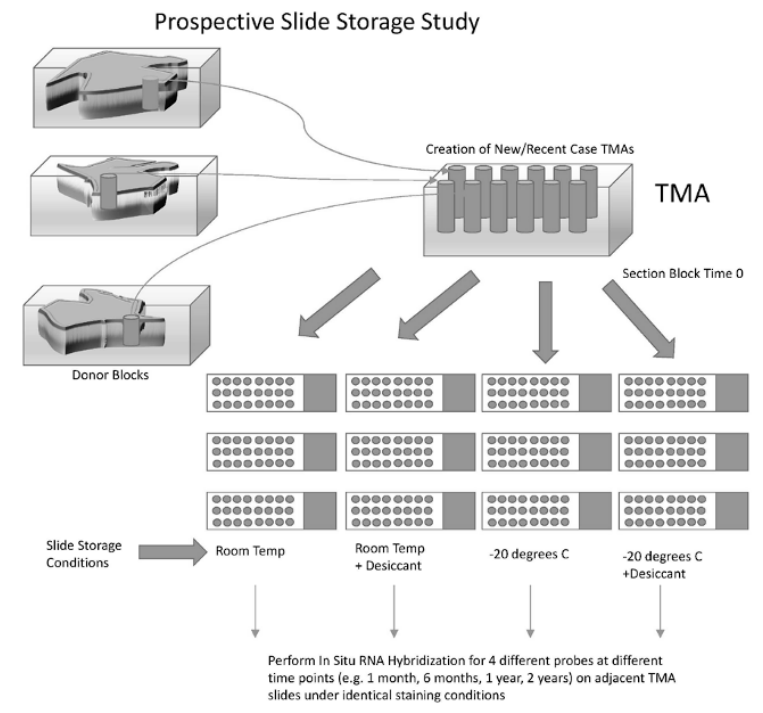

Results: There was a decrease in CISH signals in the older blocks starting at one year of age. There were lower signals that were statistically significant for each probe set when stratifying cases into 3 groups ( $<1$ year, 5-7 years, and 8-11 years). Using PDX TMAs and image analysis also showed a significant decrease of signals in relation to FFPE block age. Finally, the two-year prospective study found that $-20^{\circ} \mathrm{C}$ storage is superior to storage at room temperature.

Conclusions: Storing of FFPE tissue blocks at room temperature, the current practice in clinical and research laboratories world-wide, could lead to biased results. The standard method of FFPE block storage should be re-evaluated to better preserve the vast information that could be revealed using RISH technologies.

2114 Pathologists' Accuracy and Reproducibility Rates for PD-L1 Immune Cell Quantitation Using a Digital Pathology Image Analysis Algorithm in Urothelial Carcinoma Samples

Michael Barnes, Isaac Bai, Kien Nguyen, Joerg Bredno, Rachel Fonstad, Suresh Agarwal, Suhas Patil, Bharathi Vennapusa, June Clements, Carol Jones, Sutini Ngadiman, Azita Djalilvand, Tracie Gardner, Charles Bechert, Christoph Guetter. Roche/Ventana Medical Systems, Mountain View, CA.

Background: Atezolizumab has shown clinical efficacy in urothelial carcinoma (UC) patients and is associated with a complimentary immunohistochemistry (IHC) assay. This assay has demonstrated the predictive value of PD-L1 (SP142) IHC expression on immune cells (IC); however IC assessment is novel and pathologists' reproducibility may be a challenge. Digital pathology (DP) with image analysis (IA) has the potential to increase pathologists' precision; however, legacy implementation of clinical IA methods have used field(s)-of-view (FOV) producing mixed results. To address these issues, we developed a PD-L1 IA UC digital algorithm using a novel whole tumor (WT) approach. Design: Two cohorts of slides derived from formalin-fixed paraffin-embedded UC patient samples (60 and 76 cases) were stained for PD-L1 IHC (SP142). Ground truth accuracy for each case was determined by a consensus panel of three independent reading pathologists. Eight pathologists underwent training for IHC-based manual glass slide (MR) assay and IA interpretation for both FOV and WT approaches. Either two or three scorers independently and blindly scored each case using MR, digital read (DR, no IA but digital assessment), FOV IA, and WT IA. Data was analyzed using pair-wise overall percent agreement rates (OPA) derived from assay threshold categorical bins and/or as continuous scaled quantitative scores.

Results: For inter-reader reproducibility between two pathologists, IA WT improved OPAs (98.3\% [95\% CI, 90.9-99.7\%]) over DR- $(87.7 \%$ [76.8-93.9\%]) and IA FOVapproaches $(93.1 \%$ [83.6-97.3\%]) as assessed by binary categorical bins. When assessing inter-reader reproducibility across a continuous quantitative scale $(0-100 \%)$ and between six pathologists, IA WT improved $\left(\mathrm{r}^{2}=0.99 \%, \mathrm{rpc}=2.5\right)$ over MR $\left(\mathrm{r}^{2}=0.87 \%, \mathrm{rpc}=6.8\right)$. MR $(98.1 \%[95.2-100.0 \%])$ and IA WT $(95.8 \%[92.3-97.7 \%])$ were similar in accuracy. Conclusions: IA WT showed significant inter-pathologist reproducibility improvements over IA FOV, DR, and MR assessments. Accuracy of the algorithm is still under development. These metrics are further being characterized in a larger cohort.

2115 Comparison of $3 M Y C$ Fluorescence In Situ Hybridisation Probes and Manual versus Semi-Automatic Staining Methods; a Tissue Microarray-Based Study on $\mathbf{5 7}$ Lymphomas

James Boers, Ed Schuuring. Isala Hospital, Zwolle, Netherlands; University Medical Center Groningen, Groningen, Netherlands.

Background: MYC Fluorescence in situ hybridisation [FISH] translocation testing on every newly diagnosed diffuse large cell lymphoma [DLBCL] have become mandatory in the Netherlands. Lengthy analist handson time of FISH staining may preclude implementation of MYC FISH and warrants investigation of semiautomatic staining methods potentially resulting in substantial cost savings. New MYC FISH probes have available requiring validation before considering implementation.
Design: A tissue microarray [TMA[ was constructed of paraffin blocks of 57 lymphomas (10 Burkitt lymphomas [BL] and 47 diffuse large cell lymphomas [DLBCL]; 19 previously tested for MYC) and stained with: (1) Abbott probe, manual method; (2) Zytovision, manual; (3) Leica Kreatech probe, Leica Kreatech ThermoBrite Elite FISH robot. (1) was performed in the University Medical Center Groningen [UMCG] with substantial experience; (2)/(3) were performed in Zwolle as new tests. MYC translocation scoring was performed in Zwolle with a Leica DM6000B microscope and Cytovision software assessing at least 100 cells. Cutoff levels of normal translocation rates were determined by counting 5 tonsils with at least 200 cells each. The Abbott method was taken as the standard. The 2 other methods were compared with Abbott. In addition, TMA-based results of all 3 probes were compared with 19 previously UMCG signed out cases on full block sections.

Results:

\begin{tabular}{|l|l|l|l|l|}
\hline MYC FISH: & $\begin{array}{l}\text { Analysable } \\
\text { cases }\end{array}$ & $\begin{array}{l}\text { Concordance } \\
\text { with Abbott }\end{array}$ & $\begin{array}{l}\text { Discordance with } \\
\text { Abbott }\end{array}$ & $\begin{array}{l}\text { Discordance with } \\
19 \text { signed out } \\
\text { cases }\end{array}$ \\
\hline Abbott & 56 & NA & NA & $2(4 \%)$ \\
\hline Zytovision & 46 & $41(89 \%)$ & $5(11 \%)$ & $3(6,5 \%)$ \\
\hline Kreatech & 46 & $46(100 \%)$ & $0(0 \%)$ & $1(2 \%)$ \\
\hline
\end{tabular}

Tissue of 10 cases were not available for Zytovision and Kreatech tests due to repeated cutting of TMA. Zytovision manual method had a $11 \%$ discordance rate ( 46 cases) with the Abbott manual method while Kreatech semi-automatic method had no discordances with Abbott. Comparison of results of the present study with 19 previously signed out cases resulted in a few discordances probably due to TMA-selection bias. Handson time was 135 min with manual method (Abbott, Zytovision) and 35 min with FISH robot (Kreatech)

Conclusions: In comparison with Abbott MYC FISH manual method, Leica Kreatech probe plus semiautomatic FISH robot method has comparable results with substantially less handson time. Zytovision method resulted in a $11 \%$ discordance rate requiring more validation.

2116 Chromogenic Detection of CRTC1-MAML2 Fusion Event in Mucoepidermoid Carcinoma Samples Using a Novel RNA ISH Assay

Betty Booker, Xiao-Ming Mindy Wang, Li-Chong Wang, Xingyong Wu, Yuling Luo, Nan Su, Emily Park, Joaquin Garcia, Robert Monroe, Xiao-Jun Ma. Advanced Cell Diagnostics, Fremont, CA; Mayo Clinic, Rochester, MN

Background: Mucoepidermoid carcinomas are the most common form of salivary gland tumors with the majority of cases resulting from a single known CRTC1-MAML2 fusion event. The CRTC1-MAML2 fusion oncogene is associated with low-grade tumors and is a well-known prognostic marker to stratify patients for therapeutic treatments. Traditionally, fusion events are detected by DNA fluorescent in situ hybridization (FISH) methods which can be laborious with complex workflows and do not provide information on the transcriptional activity of the fused gene. The BaseScopeassay, which is a new chromogenic RNA ISH platform based on the RNAscope technology, allows for the detection of a single exon-exon junction in mRNA in the tissue environment on FFPE samples. The detection of specific junctional sequences created by gene fusions with BaseScope can provide single cell level detection of the fused transcript with specific cell localization.

Design: A chromogenic BaseScope assay was performed on formalin-fixed paraffin embedded (FFPE) human mucoepidermoid cancer cell lines and tissues with or without the CRTC1-MAML2 fusion event involving the joining of exon 1 of CRTC1 and exon 2 of MAML2. Detection of the fusion exon junction event using standard light microscopy was determined using a probe targeting the novel exon-exon junction in the CRTC1-MAML2 fusion transcript as well as probes for each individual gene, HsCRTC1 and Hs-MAML2.

Results: Using the BaseScope assay, we detected RNA transcript signal for the CRTC1MAML2 junction in fusion-positive mucoepidermoid cell lines and tissues. Staining with the CRTC1-MAML2 fusion probe revealed distinct signal detection of the fusion event in the form of punctate red dots in known fusion-positive cells and no detection in known fusion-negative cells.

Conclusions: Using the BaseScope assay, we demonstrate the ability to specifically detect the CRTC1-MAML2 fusion transcripts in fusion-positive cells and tissues. This assay can easily be performed in clinical labs on routinely available FFPE samples and results can be obtained rapidly since the assay can be performed in one day and reviewed under the light microscope. In conclusion, the BaseScope assay provides an alternative chromogenic method to DNA FISH for the detection of gene fusion events in human tumor tissue samples.

2117 Comparative Evaluation of Alternative Bone Marrow Specimen Types for Targeted Next Generation Sequencing

Sarah Carroll, Molly Accola, William Rehrauer, David Yang. University of Wisconsin School of Medicine and Public Health, Madison, WI.

Background: Next generation sequencing (NGS) is increasingly being used in hematopathology to aid in characterization and classification of hematologic malignancies. The typical specimens utilized are fresh or frozen bone marrow aspirates. However, instances arise where the diagnosis of a hematologic malignancy is unexpected and the typical specimen types are not available for analysis. To date, few studies have assessed the use of specimens such as decalcified formalin-fixed paraffin embedded (dFFPE) bone marrow cores for NGS and to our knowledge, none have examined the use of extra unstained bone marrow aspirate smears. The goal of this study was to assess the adequacy of these archival specimen types for targeted NGS analysis.

Design: Eight cases with triplicate specimen types (frozen bone marrow aspirate, dFFPE bone marrow core, and unstained aspirate smear) were identified: five acute 
myeloid leukemias, two chronic lymphocytic leukemias, and one follicular lymphoma. Libraries were prepared from extracted DNA using the Ion AmpliSeq Cancer Hotspot Panel v2 and sequenced on the Ion Torrent Personal Genome Machine. The number of mapped reads, mean coverage depth, $\%$ on target reads, uniformity of coverage, number of variants identified, and variant allele frequencies from each sample type were then compared by analysis of variance.

Results: For each case, the number of variant alleles identified ranged from 13 to 25 and all variants were consistently detected across the specimen types. A significant difference in the total variant allele frequencies was not appreciated across the sample types, nor was there a significant difference in number of mapped reads, coverage depth, or $\%$ on target reads (figure 1). A difference in the uniformity of coverage was detected where the dFFPE specimens had lower uniformity than the frozen or aspirate smear specimens.

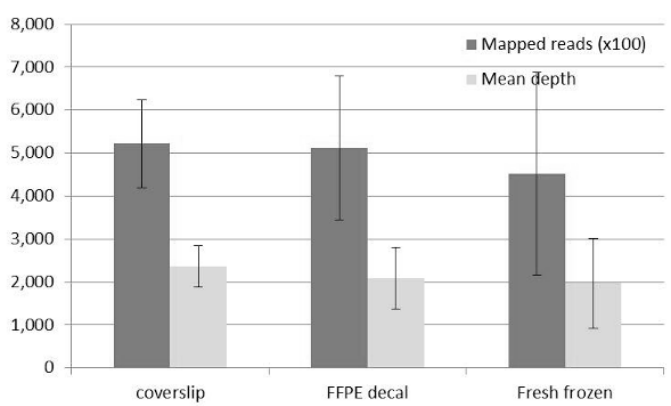

Conclusions: Analysis of triplicate specimen types shows dFFPE bone marrow core biopsies and unstained bone marrow aspirate smears are comparable to frozen bone marrow aspirate samples for detection and quantification of variant alleles on a targeted NGS panel. Unstained bone marrow aspirate smears may represent an un-tapped resource for clinical molecular diagnostics as well as correlative research.

2118 Ultrastructural Evaluation of Dendritic Cells in Light ChainMediated Acute Tubulointerstitial Nephritis

Mingyu Cheng, Jiamin Teng, Guillermo A Herrera. Louisiana State University Health Sciences Center, Shreveport, LA.

Background: Light chain-mediated acute tubulointerstitial nephritis (LC-ATIN) is an unusual inflammatory renal disease in patients with plasma cell dyscrasia. Previous studies showed that accumulation of light chain along tubular basement membranes (TBM) may directly damage the proximal tubular epithelial cells by overloading the lysosomal system. Dendritic cells (DCs) play a critical role in the regulation of the adaptive immune system. However, their involvement in LC-ATIN is still unknown. In this study, the ultrastructural features of DCs in LC-ATIN were evaluated with transmission electron microscopy (TEM) and immunogold labeling.

Design: Nine cases of LC-ATIN were identified retrospectively from the archives of renal biopsy specimens previously evaluated in the Department of Pathology at LSUHSC-Shreveport from 2013 through 2015. All biopsy specimens were processed for TEM according to standard techniques. Anti-CD1c antibody was used for ultrastructural immunogold labeling of DCs in renal biopsy specimens.

Results: Ultrastructually, the DCs in renal biopsies were identified by their characteristic dendritic morphology: indented nuclei with a peripheral distribution of heterochromatin, an abundance of cytoplasmic vesicles, and blunt, elongated dendritic processes. In renal biopsies with LC-ATIN, DCs were observed to have direct contact with TBM and lymphocytes. Immunogold labeling showed multiple fragments of CD1c positive DCs attached to the TBM in LC-ATIN.

Conclusions: The ultrastructural finding of direct contact between DCs and TBM in renal biopsies with LC-ATIN, which was confirmed by immunogold labeling, suggests that DCs may participate in the inflammatory process of LC-ATIN by surveying the local environment for signs of damage. The finding of direct contact between DCs and lymphocytes suggests that intra-renal antigen presentation by DCs to lymphocytes, which rarely happens in peripheral tissue, may be involved in the immune responses in the kidney in patients with LC-ATIN

2119 Sensitive and Specific Detection of Mutations in Circulating Tumor DNA with Anchored Multiplex PCR and Next-Generation Sequencing Brady P Culver, Jerome E Lee, Josh Haimes, Namitha Manoj, Eric M Davis, Ian McKittrick, Katelyn E Trifilo, Laura A Johnson, Laura Griffin, Brian Kudlow. ArcherDX, Inc., Boulder, $\mathrm{CO}$.

Background: Liquid biopsies are a promising, minimally invasive alternative to solid tumor biopsies that have potential cost, time and safety benefits, as well asa greater ability to interrogate heterogeneous tumors. However, except in advanced disease states, cell free DNA (cfDNA) is typically of low abundance and only a small portion of cfDNA originates from tumor cells as circulating tumor DNA (ctDNA), which tends to be highly fragmented (100-300bp). Therefore, NGS-based assays to detect variants in ctDNA must be sensitive enough to detect mutations at allele frequencies (AF) $<2 \%$ from $<100$ ng of highly fragmented DNA.

Design: We developed the Archer ${ }^{\circledR}$ Reveal ctDNA ${ }^{\mathrm{TM}} 28$ assay based on Anchored Multiplex PCR (AMPTM), a target enrichment method for NGS that uses unidirectional gene-specific primers and molecular barcoded (MBC) adapters for amplification. This enables preferential enrichment of small, degraded ctDNA fragments over long genomic DNA fragments, thereby increasing detection sensitivity of mutations in ctDNA by NGS. $\mathrm{MBC}$ adapters ligated prior to amplification permit post-sequencing error correction, reducing background noise and increasing analytical sensitivity of detecting ultra low-allele frequency variants. Variant filtering in the Archer Analysis pipeline further increases the specificity of variant calls.

Results: Using commercially available synthetic ctDNA standards, we demonstrate that the AMP-based Reveal ctDNA 28 assay preferentially enriches small, fragmented ctDNA over genomic DNA. Based on sequenced reads, AMP enabled interrogation of more than $65 \%$ of the input molecules from 50ng starting material. As a result, we show $100 \%$ detection sensitivity for $1 \% \mathrm{AF}$ variants using 10ng DNA input and $71.9 \%$ detection sensitivity for $0.1 \% \mathrm{AF}$ variants using 50ng DNA input. MBC-enabled post-sequencing error correction and variant filtering reduced the number of false positives by $98 \%$, resulting in $91.7 \%$ specificity. Finally, Reveal ctDNA 28 accurately detected mutations present in corresponding tissue biopsies with under 10ng liquid biopsy-derived DNA. Conclusions: The Archer Reveal ctDNA 28 assay is a powerful tool for sensitive and specific NGS-based detection of variants in reference ctDNA standards and shows promise for accurate detection of variants in ctDNA from clinical liquid biopsies.

2120 InDelDuper: A Highly Sensitive and Specific Algorithm for FLT3 ITD Detection in Next-Generation Sequencing Data

Yi Ding, William Crowe, Todd Laughlin, John Spence, Jan Spence, Paul G Rothberg. Richard Burack. University of Rochester Medical Center, Rochester, NY.

Background: Internal tandem duplication (ITD) in the fms-related tyrosine kinase 3 gene (FLT3) is a recurrent somatic mutation frequently found in cytogenetically normal acute myeloid leukemia (AML). The FLT3 ITD mutation strongly correlates with lower overall survival in AML patients. Although recent advances in high-throughput sequencing technologies have enabled genome-wide detection of various genomic alterations in cancer cells, detection of insertions in short read Next-Generation Sequencing (NGS) data is a challenge because insert-containing reads often fail to align to the reference genome.

Design: Eighty-five AML samples positive for various mutations as detected by prior sequencing were selected for study. Sixteen of these samples were known positive for FLT3 ITD with lengths ranging from 21 to 220 base-pairs (bp) as determined by electrophoresis. To improve the ability to detect large insertions, we used MiSeq $\mathbb{R}$ Reagent Kit V3, which permitted 2x301 bp read lengths. The Illumina MiSeq Reporter software can only detect short insertions and deletions, which causes a large fraction of the FLT3-ITDs to be undetectable. To address this deficit, we developed a customized pipeline, Insertion-Deletion Detection Using Paired End Reads (InDelDuper). We compared the performance of InDelDuper with two other variant callers that have utility for detecting indels, VarDict and Pindel.

Results: InDelDuper was evaluated on 85 AML samples, of which 16 were positive for FLT3 ITD mutations with 2 samples containing two distinct ITDs. We compared results of InDelDuper to VarDict and Pindel. InDelDuper detected 100\% (18 of 18) of the FLT3 ITDs with no false positives. It outperformed the other two programs. Pindel (version 0.2 .4 ) was able to detect 11 of 18 ITDs (61\% sensitivity) and VarDict (version b1) detected 15 of 18 ITDs ( $83 \%$ sensitivity). In addition, InDelDuper provided a precise definition of the duplication start-site, in contrast to both Pindel and VarDict which provided a range of duplication start locations.

Conclusions: InDelDuper is a very sensitive and specific tool which can detect partial, large and complex tandem duplications. It can also provide a precise location of the duplication start site. InDelDuper can be readily incorporated into nearly any analysis pipeline for somatic mutation detection in paired-end amplicon-based NGS data.

\section{Characterization of B- and T-cell Immune Repertoires with} Anchored Multiplex PCR and Next-Generation Sequencing

Jens Eberlein, Thomas Harrison, Ian McKittrick, Megan Wemmer, Laura Griffin, Brady P Culver, Laura A Johnson, Brian Kudlow. ArcherDX, Inc., Boulder, CO.

Background: The adaptive immune system is involved in various disease conditions including cancer, chronic infection, autoimmune disease and transplant rejection. Adaptive immunity is mediated by B and T lymphocytes, which are activated upon antigen binding to antigen receptors expressed on their surface. Therefore, the spectrum of these antigen receptors, or immune repertoire (IR), provides a means to monitor adaptive immune responses to disease, vaccination and therapeutic interventions. Nextgeneration sequencing (NGS) of antigen receptor genes is a valuable tool in the study of disease states and responses to various interventions. Traditional amplicon-based NGS assays use opposing primers for targeted amplification of rearranged antigen receptor genes. Thus, large primer panels are required to capture the extensive combinatorial diversity exhibited by the IR. Quantification from such assays requires a complex system of synthetic controls to account for differential amplification efficiency across segment combinations. Here, we describe an Anchored Multiplex PCR (AMPTM)-based NGS assay to analyze the IR, employing a minimal set of gene-specific primers in conjunction with molecular barcodes (MBCs) to reduce amplification bias.

Design: AMP uses MBCs ligated to cDNA ends and gene-specific primers for amplification, enabling immune chain mRNA interrogation from a single side. This eliminates the need for opposing primers that bind within the highly variable V-segment, eliminating clone dropout due to somatic hypermutation. Furthermore, this facilitates CDR3 sequence capture from highly fragmented RNA inputs. We validated the quantitative reproducibility and sensitivity of AMP-based B- and T-cell IR assays using mRNA isolated from peripheral blood leukocytes.

Results: Our data showed high reproducibility between replicates and quantitative clone tracking down to $0.01 \%$, with the ability to determine IGHV mutational status. Furthermore, our data indicate that clonal diversity in sequencing data is driven by input quantity and total T-cell number.

Conclusions: AMP-based NGS with MBC quantification and error-correction is a powerful method to characterize the immune repertoire. This enables sensitive clone tracking and measurement of lymphocyte diversity from clinical RNA samples. 
2122 Detection of FGFR1-PLAG1 Fusion in Salivary Carcinomas Ex Pleomorphic Adenoma by Fluorescence In Situ Hybridization and Next Generation Sequencing

Soufiane El Hallani, Simon Chiosea. University of Pittsburgh, Pittsburgh, PA.

Background: One of the molecular hallmarks of pleomorphic adenoma (PA) and carcinomas ex PA are fusions involving Pleomorphic adenoma gene 1 (PLAG1; 8q12). Several PLAG1 fusion partners are known, including Fibroblast growth factor receptor 1 (FGFRI; 8p11). We compared the ability of the more commonly used fluorescence in situ hybridization (FISH) technique and a next generation sequencing (NGS)-based fusion detection assay to detect intrachromosomal FGFRI-PLAGl in carcinomas ex-PA. Design: Formalin fixed paraffin embedded samples of 22 salivary carcinomas were tested for PLAGI rearrangements by a commercial PLAGI break-apart FISH probe that may detect rearrangement regardless of the fusion partner (PMID: 27379604) and a laboratory developed multiplex fusion assay that would detect intrachromosomal exon 2 FGFR1-exon 2 PLAG1 fusion (PMID: 26747586). Eleven epithelial myoepithelial carcinomas (EMCA) and 10 salivary duct carcinomas (SDC) were tested. Results: Results are summarized in Table 1.

\begin{tabular}{|c|l|c|c|}
\hline \multicolumn{2}{|c|}{} & \multicolumn{2}{c|}{ FGFR1-PLAG1 NGS-based fusion assay } \\
\cline { 3 - 4 } \multicolumn{2}{|c|}{} & Positive & Negative \\
\hline \multirow{2}{*}{ PLAG1 FISH } & Positive & 1 & 5 \\
\cline { 2 - 4 } & Negative & 2 & 14 \\
\hline
\end{tabular}

NGS-based fusion assay identified 3 FGFR1-PLAG1 positive cases, in 2 of which PLAG1 rearrangement was not identified by FISH.

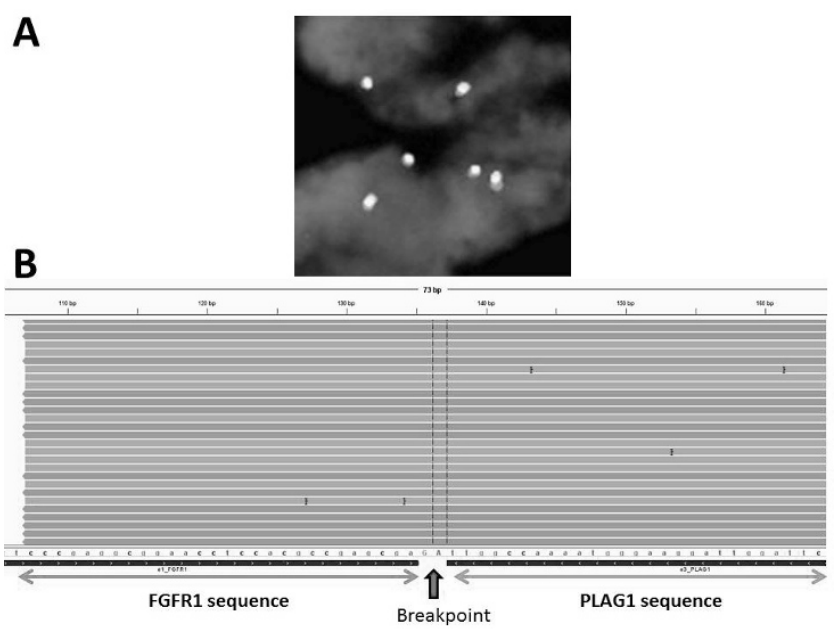

Figure 1A shows PLAG1 FISH (intact yellow signal without the green/red signal split) in an FGFR1-PLAG1 positive EMCA. Figure 1B shows the read alignments spanning both fusion partners (PLAGI and FGFRI) and crossing the breakpoint. The one SDC case with NGS-proven FGFR1-PLAG1 fusion was called positive by PLAG1 FISH due to the concomitant presence of chromosome 8 hyperploidy or PLAG1 amplification in $>75 \%$ of cells. PLAG1 FISH was positive in 6 cases, in 5 of which FGFR1-PLAG1 fusion was not identified by NGS-based fusion assay, probably due to the rearragement of PLAG1 with a gene partner other than FGFRI.

Conclusions: PLAG1 break-apart FISH does not identify intrachromosomal FGFR1PLAG1 fusions that are not accompanied by chromosome 8 hyperploidy or PLAGI amplification. Therefore, PLAGI break-apart FISH underestimates the prevalence of salivary carcinomas arising ex PA by about $10 \%$.

2123 DDX4 - A Novel Immunohistochemical Marker for Immature Germ Cells and a Valuable Tool for Infertility Testicular Biopsy Evaluation Nazneen Fatima, Rajen Goyal, Lu Wang, Robert E Brannigan, Ximing J Yang. Northwestern Memorial Hospital, Chicago, IL.

Background: Testicular biopsy is routinely performed in men with infertility to evaluate the testicular pathology, particularly the presence or absence of germ cells. Hypospermatogenesis can be caused by several different factors which can be corrected medically or may necessitate the sperm retrieval. However, for Sertoli-cell only syndrome no effective treatment is available to reverse the condition and sperm retrieval is not an option. An immunohistochemical (IHC) marker would be of great value to highlight the immature germ cells in testicular biopsy and guide the management. We report the utility of a novel germ cell marker DDX4 (DEAD-box helicase 4) in the infertility testicular biopsy evaluation.

Design: Testicular biopsies $(n=40)$ and orchiectomy specimens $(n=20)$ were examined and assessed with specific attention to germ cell identification, distribution, and maturation patterns. They were subsequently categorized into the following patterns: Sertoli cell-only morphology, germ cell sloughing, and maturation arrest. IHC were performed with an antibody specific for DDX4.

Results: The DDX4 typically shows strong cytoplasmic staining in immature germ cells (spermatogonia and spermatocytes), weak staining in spermatids, and negative staining in mature spermatozoa (sperms). Sertoli cells and Leydig cells are negative for DDX4. Three of 14 originally designated Sertoli cell-only morphology specimens were found to have rare immature germ cells in the tubules by DDX4 staining. Germ cell sloughing cases often show disorganized DDX4 positive immature germ cells in center and periphery of the seminiferous tubules. In maturation arrest group, DDX4 staining highlighted a reduced number of immature germ cells.

Conclusions: DDX4, a novel biomarker, is highly sensitive and specific marker for immature germ cells. It is a useful tool for quantitative analysis of infertility testicular biopsy cases. Highlighting the rare immature germ cells in hypospermatogenesis cases and separating it from sertoli-cell only pattern, can offer better management of these patient and improved clinical outcome.

2124 RNA In Situ Hybridization Using Quantigene ViewRNA 2.0 Branched DNA Signal Amplification Technology Shows PGC1B and ESRRA Are Transcriptionally Upregulated Late in Colon Cancer Development

Kurt Fisher, Binita Das, Beth Clymer, Geoffrey Talmon, Robert Lewis. University of Nebraska Medical Center, Omaha, NE.

Background: We have shown that transcriptional activators peroxisome proliferatoractivated receptor gamma, coactivator 1 beta (PGC1B) and estrogen-related receptor alpha (ESRRA) are aberrantly upregulated at the mRNA level in human colorectal cancer metastases and promote the survival of cancer cells. We designed the current study to assess the levels of PGC1B and ESRRA in earlier stages of colorectal cancer development.

Design: Using $2 \mathrm{~mm}$ tissue microarrays (TMAs) of formalin fixed paraffin embedded tissue we quantitatively assessed the mRNA levels of PGC1B and ESRRA using Quantigene ViewRNA 2.0 technology in 14 normal colon, 3 tubular adenomas, $5 \mathrm{pT} 1$ stage adenocarcinomas, 4 pT2 stage adenocarcinomas. The TMAs were pretreated then probed with either a non-specific probe set or a mixture of probes for PGC1B and ESRRA at $40^{\circ} \mathrm{C}$ for 2 hours then washed in wash buffer. The following day, the TMAs were hybridized to preamplifier solution, amplifier solution, label probe and the signal was developed using fast red substrate and counterstain for DAPI following the manufacturer's protocol. Total signal for each gene was counted using a fluorescent microscope with the appropriate filters and assessed by unit area and nuclei count for three regions within each individual TMA spot, with three separate biological replicates from the same case.

Results: We successfully generated fluorescent signals for two different RNA probes from TMAs with low background signal. PGC1B and ESSRA are expressed in normal colonic epithelium, and do not appear to have significant expression in hematopoietic or stromal cells. PGC1B and ESSRA are significantly increased only in pT2 tumors compared to normal controls ( $\mathrm{p}$-value $<0.05$ ).

Conclusions: 1) PGC1B and ESSRA mRNA is upregulated in later stage colorectal adenocarcinomas, but not in tubular adenomas and pT1 tumors, suggesting these genes are required for metastatic progression, but not tumor initiation.

2) Variables associated with high background include, but are not limited to: 1) Insufficient wash time 2) Extracellular mucinous debris 3) Suboptimal filtering of fluorescent channels.

3) Colorimetric signal analysis is best reserved for highly expressed genes and potentially limited to assessment of mRNA cell localization, while fluorescent signals can be quantitated, even for lowly expressed genes.

\section{Improving Diagnostic Accuracy in the Pathology Workflow by} Integration of Raman Microscopy

Andrée-Anne Grosset, Catherine St-Pierre, Michael Jermyn, Kelly Aubertin, André Kougioumoutzakis, Marion Pineau, Karl St-Arnaud, Maria C Vladoiu, Frédéric Leblond, Dominique Trudel. Centre de Recherche du Centre Hospitalier de l'Université de Montréal (CRCHUM), Montreal, QC, Canada; Université de Montréal, Montreal, QC, Canada; Polytechnique Montreal, Montreal, QC, Canada; Dartmouth College, Hanover, NH; Centre Hospitalier de l'Université de Montréal (CHUM), Montreal, QC, Canada. Background: The development of complementary analyses to assist pathologists resolving diagnostic uncertainties is a constant need. Raman microscopy is a labelfree molecular imaging technique that uses backscattered light following tissue laser excitation, to indicate the molecular content of the tissue specimen. However, this technique is very sensitive to glass and to chemicals used to process formalin-fixed paraffin-embedded (FFPE) tissues, which greatly limits its efficacy.

Design: We developed a rapid standardized protocol for the preparation of FFPE diagnostic tissues, providing rich molecular content by Raman imaging that can be exploited for clinical use. The preparation involved $4 \mu \mathrm{m}$ tissue sections on low cost aluminum slides, dewaxing in 8 minutes, and 20 minutes of drying. All Raman spectra were acquired with a confocal Raman microscope.

Results: A total of 135 Raman spectra from 16 patients were analysed from a prostate cancer (PCa) tissue microarray ( 8 benign, 5 tumors with a Gleason Score (GS) of $3+3$, and 3 tumors with a GS of 4+4). Using the Support Vector Machine classifier, our results with Raman microscopy identified the tumor grade of PCa tissue with an accuracy of $91 \%$, a sensitivity of $92 \%$ and a specificity of $87 \%$.

Conclusions: Raman microscopy signatures of PCa tissue demonstrate the potential to improve current pathology practices with clinical impact. Therefore, this technology could decrease the number of subsequent biopsies and radical prostatectomy, which are expensive and time-consuming. 
2126 Glomerular Endothelial Cells Contribute to Circulating Endothelial Microparticles in Patients with Diabetic Glomerulopathy

Xin Gu, Jehan A Abdulsattar, Guillermo A Herrera. LSUHSC-Shreveport, Shreveport, LA.

Background: Endothelial microparticles (EMP) are small vesicles released from disturbed endothelial cells (EC). Circulating EMP in plasm is used as a marker of EC disturbance. Previous clinical studies have revealed that plasma levels of EMP were elevated in many diseases include diabetes mellitus. However, wether glomerular endothelial injury contributes to circulating EMP, and wether elevated plasm EMP level can be used to as a marker for kidney endothelial dysfunction in diabetic patients are unclear. The aim of this study is using ultrastructural morphologic method to exam EMP in diabetic glomerulopathy (DG).

Design: Thirty kidney biopsies, fifteen with DG and fifteen with minimal change disease (MCD), were selected from archived materials. Following examination of thin-sections and confirmation of diagnosis, ultrathin sections were scoped under transmission electron microscopy. In each case, one glomerulus was selected and four cross sections of glomerular loops were examined. Endothelial morphology is examined and the number of EMP within the loops was counted.

Results: In MCD group, the endothelial cells were smooth and less than 10\% loops (5) contained EMPs that measured from 0.1 to $0.5 \mu \mathrm{m}$ (figure 1).

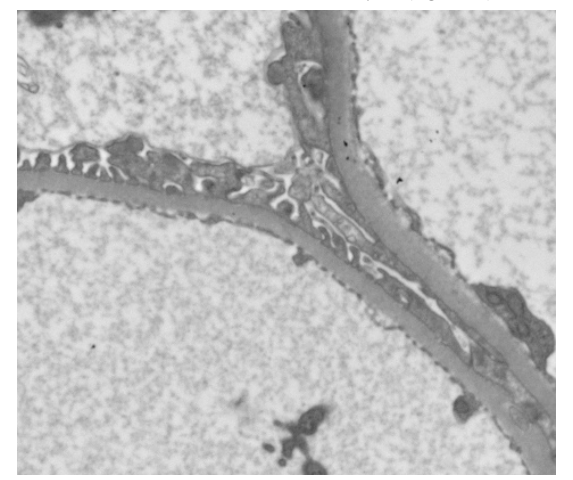

In DG group, up to $40 \%$ loops showed irregularity of endothelial lining, protruding and tufting of endothelial membranes or segmental lifting from basement membranes. EMPs were identified in 30\% (18) loops (figure 2, arrows indicate EMPs).

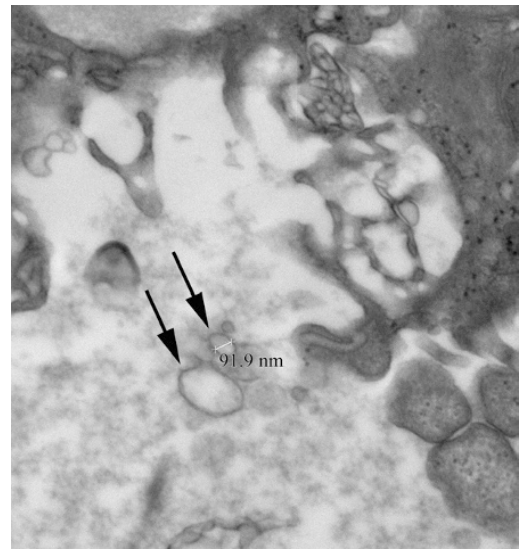

Conclusions: Ultrastructural features of endothelial injury present in DG and EMP can be identified by transmission electron microscopy examination of renal biopsies. Compared with MCD, EMP is significant higher in DG. These results suggested that, in diabetic patients, hyperglycemia induced glomerular endothelial injury contribute to elevated plasma EMP.

\section{Immunohistochemistry in Lymphomas with Crush Artifacts}

Monira Haque, Jacek Polski. University of South Alabama, Mobile, AL

Background: Crush artifacts are common in surgical specimens and small biopsies in particular. Lymphoid cells are prone to being crushed producing unidentifiable cellular details, which can create great diagnostic difficulties. To assess the diagnostic utility of immunohistochemistry (IHC) in lymphomas with crush artifacts, we evaluated performance of IHC in crushed lymphoma tissues.

Design: Pathology archives were searched for lymphomas with crush artifacts. Cases were reviewed to determine if both crushed and non-crushed lymphoma tissues were subjected to IHC during diagnostic work-up. Qualitative (positive/negative) and quantitative scoring (H-score) was performed by a hematopathologist for both crushed and non-crushed areas in each case. Spearman's rank correlation coefficient was used to determine correlations between $\mathrm{H}$-scores.

Results: 19 cases qualified for the study. IHC included multiple lymphoid and nonlymphoid markers. A very strong positive correlation was shown between all $\mathrm{H}$-scores for crushed versus non-crushed tissue $(\mathrm{r}=0.87, \mathrm{p}<0.000001, \mathrm{n}=132)$. A very strong correlation was also observed for Bcl-2 $(\mathrm{r}=0.99)$ and CD45 $(\mathrm{r}=0.91)$. The correlation was strong for $\mathrm{CD} 3(\mathrm{r}=0.77)$ and $\mathrm{CD} 5(\mathrm{r}=0.75)$. Moderate correlation was observed for Bcl-6 (r=0.59) and CD20 (r=0.57).
There was also good agreement for qualitative scoring of crushed tissue versus non-crushed tissue with full agreement in $86.3 \%$ of all IHC stains. Agreement was excellent for CD20 (100\%), Bcl-6 (100\%), Ki-67 (100\%) and Cam5.2 (100\%); good for $\mathrm{CD} 45(87.5 \%), \mathrm{CD} 5(85.7 \%)$, and Bcl-2 (85.7\%). Markers that showed qualitative discordance between crushed and non-crushed tissue were CD3, CD10, CD5, CD45, AE1/AE3, Bcl-2, Lambda, MUM1, p63, and Vimentin. The qualitative discordance was more common due to false positive rather than false negative results. Discordance was frequently observed for CD3 (40\%) and CD10 (33.3\%), and in both cases consisted of false positive staining in crushed tissues. The false positive qualitative results for CD3, $\mathrm{CD} 5$, and CD45 were caused by inability to distinguish numerous reactive T-cells from lymphoma cells in crushed areas.

Conclusions: The data shows overall good correlation and agreement between IHC of crushed versus non-crushed lymphoma tissue. This suggests that lymphoma cases with crush artifacts are still amenable to IHC analysis. Even though the H-score correlation for $\mathrm{CD} 20$ was found to be moderate, qualitative scoring showed $100 \%$ agreement between crushed and non-crushed tissues. We conclude that strong, diffuse staining of crushed tissue for CD20 correctly predicts B-cell lineage of the lymphoma cells.

\section{Novel Herceptin-Based IHC Test Detects HER2+ Breast Cancers} Correlating to Responses to HER2-Targeted Therapy

Jian He, Zhiqing Zhang, Shuo Chen, Angelina Motiee, Yonghua Zhang, Songqing Zhao, Jianfu Wang, Constance T Albarracin, Susan Abraham, Yun Wu. UT MD Anderson Cancer Center, Houston, TX; Novodiax, Inc., Haywood, CA.

Background: HER2+breast cancer is an aggressive subtype that accounts for $15-20 \%$ of breast cancers. HER2-targeted therapy with trastuzumab (Herceptin) has significantly improved the survival of patients with both primary and metastatic HER2+ breast cancer. Current ASCO/CAP breast cancer therapy guidelines require HER2 tests by immunohistochemistry (IHC) and/or in situ hybridization (ISH). However, more than $50 \%$ of HER2 + patients identified by those tests develop de novo or acquired resistance to Herceptin therapy. A possible explanation for Herceptin treatment failure is that the current HER 2 tests only identify tumors with HER2 protein overexpression or gene amplification, but they do not detect whether trastuzumab, a HER2-targeted humanized monoclonal therapeutic antibody, can specifically bind to HER2 proteins of breast cancers. Novodiax has developed a novel Herceptin-based IHC test (ihcDirect-Herceptin) which can directly detect trastuzumab binding to breast cancer. We hypothesize that Herceptin-based IHC will predict which HER2+ breast cancers respond to trastuzumab therapy.

Design: The ihcDirect-Herceptin test was developed by linking Herceptin (trastuzumab) to a unique polymerized horseradish peroxidase (HRP). 23 cases of HER2+ breast cancer patients (verified by ASCO/CAP-recommended IHC and/or FISH tests) were selected for the study. All 23 HER2+ breast cancer patients received neoadjuvant or adjuvant transtuzumab therapy. One 4-umunstained slide from each case was stained by ihcDirect-Herceptin test. Staining results were then correlated with responses to trastuzumab therapy. Response to trastuzumab was defined as no residual invasive carcinoma in the breast and lymph node post neoadjuvant trastuzumab therapy, or no recurrence after adjuvant trastuzumab therapy for more than 5 years.

Results: Twelve (52\%) of 23 HER2 + breast cancers were also positive for ihcDirectHerceptin test, and $8(67 \%)$ of these 12 patients showed good response to trastuzumab therapy. In contrast, only $2(18 \%)$ of 11 patients whose tumors were HER $2+$ but negative by ihcDirect-Herceptin test had a good response to trastuzumab therapy $(\mathrm{p}=0.019$, Fisher exact test) (Table 1).

\begin{tabular}{|l|l|l|l|}
\hline & \multicolumn{3}{|l|}{ ihcDirect-Herceptin } \\
\hline Response to trastuzumab treatment & + & - & Total \\
\hline good & 8 & 2 & 10 \\
\hline poor & 4 & 9 & 13 \\
\hline total & 12 & 11 & 23 \\
\hline
\end{tabular}

Conclusions: Our results indicate that ihcDirect-Herceptin test is positive in approximately half of HER $2+$ breast cancers, and that positivity by ihcDirect-Herceptin significantly correlates with clinical response to trastuzumab therapy.

\section{Assessing the Clinical Utility of Expanded vs. Small Targeted Solid Tumor NGS Panels}

Amin A Hedayat, Jason D Peterson, Julie Y Tse, Cheryl L Eifert, Torrey L Gallagher, Sophie J Deharvengt, Francine B de Abreu, Gregory J Tsongalis. Geisel School of Medicine at Dartmouth, Hanover, NH; Dartmouth Hitchcock Medical Center and Norris Cotton Cancer Center, Lebanon, NH; KEW, Inc., Cambridge, MA.

Background: Next Generation Sequencing (NGS) has become an essential tool in the personalized treatment of solid tumors. Multi-gene panels are routinely used to identify clinically actionable mutations in tumor samples, and determine clinical trial eligibility. When compared to small targeted panels, expanded panels can detect additional actionable mutations providing a comprehensive therapeutic and prognostic molecular profile. Incorporating further molecular information (i.e. MSI, CNV, Mutation Burden-Immune Oncology) can increase actionable mutation detection rates. We aimed to evaluate the clinical utility of an expanded panel in identifying actionable mutations in samples previously characterized as wildtype using a smaller targeted panel.

Design: Twelve solid tumor specimens (4 colorectal carcinomas, 8 CNS tumors) previously characterized as wildtype by a 50 -gene targeted NGS Panel (Ampliseq CHPv2) were sequenced using an expanded NGS panel (CANCERPLEX FP+IO Kew, Inc.) which included MSI and IO (colorectal carcinomas) and TERT promoter mutations (Glioblastomas).

Results: Molecular alterations were identified in $75.0 \%$ of the samples ( 9 of 12 ). A total of 30 mutations were identified (10 SNVs/INDELs and $20 \mathrm{CNVs})$. None of the 
30 mutations had associated FDA approved therapies for the indicated tumor type, however FDA approved therapies in other cancer types were identified for $>76.0 \%$ of the mutations. Applicable clinical trials were also identified in all mutated samples ( 9 of 12). In addition, TERT promoter mutations were detected in $33.0 \%$ of the glioblastoma samples.

Conclusions: Expanded NGS panels are capable of detecting potentially actionable mutations in patient samples that would be characterized as wildtype using a smaller targeted panel. Generating an expanded molecular tumor profile increases both the number of available treatment options as well as the likelihood of identifying an applicable clinical trial, which can ultimately lead to improved patient outcomes. In addition, including other tumor profiling techniques, such as MSI and IO to assess response to immune checkpoint inhibitors, into a single expanded NGS panel can reduce result turn-around-times and testing costs by decreasing the number of separate molecular tests that must be performed on a specimen.

\section{Solvent Fee Environmentally Friendly IHC}

Alfonso Heras, Chase V Furler, Carolyn M Wall. BIO SB, Inc., Santa Barbara, CA. Background: The current deparaffinization, heat epitope retrieval and mounting of tissue biopsies for IHC rely on the removal of paraffin or the mounting of specimens with toxic, flammable solvents. Current heat assisted 3-in-1 one-step deparaffinization solutions leave paraffin leftovers, which need to be eliminated with solvents prior to permanent mounting. We report on the utilization of an environmentally friendly onestep heat-assisted deparaffinization, heat retrieval and hydration procedure, plus the mounting of tissue specimens with ChromoProtector and a non-toxic mounting solution. Design: Normal 23-core TMA's and FFPE cancer tissues were subjected to the IHC of CD20, CK AE1/AE3 and PR were detected with PolyDetector HRP/DAB using the TintoStainer automated system. Xylene/alcohol deparaffinization and Citrate HIER was used as control. The TintoDeparaffinator Citrate and EDTA were compared to the 3 in 1 Trilogy and Dewax and HIER Buffer Low and High. Tissues were used with all the experimental deparaffinization solutions in a pressure cooker at $121^{\circ} \mathrm{C}$ for 15 min or a PT Module at $98^{\circ} \mathrm{C}$ for $30 \mathrm{~min}$. All deparaffinization solutions were mounted with or without ChromoProtector and mounted with AquaMounter, xylene containing PermaMounter, or XyGreen PermaMounter, a biodegradable permanent mounting media. Specificity and sensitivity results were graded by 3 highly trained personnel using a $0-4$ signal and background scale. A T-test was used to evaluate statistically significant differences.

Results: All the deparaffinization solutions produced similar acceptable results, but had an evident micro-paraffin leftover at high magnification when mounted with AquaMounter. The TDP Citrate and TDP EDTA had significantly less micro-paraffin leftover than Trilogy and Dewax HIER Buffer. When all 3-in-1deparaffinization experimental tissues were treated with ChromoProtector prior to AquaMounter, PermaMounter or XyGreen PermaMounter, no micro-paraffin leftover was observed. The elimination of solvents for deparaffinization and mounting reduced the procedure time by 1 hour.

Conclusions: When IHC procedures are performed using the TDP to deparaffinize and retrieve FFPE tissues, the ChromoProtector to preserve stains and XyGreen PermaMounter to permanent mount tissues, allow for a solvent-free environment when conducting IHC procedures. These solutions are a safe, efficient and economical alternatives to traditional deparaffinization and mounting of tissues. An additional benefit is the reduction of cost, time and number of steps in the deparaffinization, hydration and epitope retrieval of tissues and the exposure to toxic solvents when handling FFPE tissues for IHC.

\section{Predictive Cytologic Topography Improves Detection of High-} Grade Prostate Cancer on MRI

Ken A Iczkowski, Amy Kaczmarowski, Sarah L Hurrell, Anjishnu Banerjee, Kenneth Jacobsohn, Sean McGarry, Mark Hohenwalter, Peter S LaViolette. Medical College of Wisconsin, Milwaukee, WI

Background: Radiologic-pathologic correlation has begun to allow the validation of prostate cancer imaging technology. More accurate imaging can be created using the combination of these modalities to train machine learning algorithms for improved non-invasive detection of prostate cancer.

Design: Thirty-two prostatectomy patients were imaged $\leq 2$ weeks pre-operatively using a 3T MRI (GE) and quantitative diffusion-weighted imaging. Custom 3D-printed slicing molds were made for each tissue to match the MRI orientation and slice thickness. Scanned whole-mount slides were digitally segmented into lumen, stroma, and epithelium using custom Matlab code. Digitized histology was co-registered to T2-weighted MRI using Matlab's non-linear warping, then used to train a partial least squares regression algorithm. A leave one out approach was used to generate Predictive Cytologic Topography (PiCT) maps of histologic features for each patient using data from the other 31 patients. PiCT maps were then compared to urologic pathologistannotated areas color-coded by Gleason (G) grades and to radiologist-graded PI-RADS version 2 lesions of score $4+$ (out of $5+; 4+=$ high likelihood of cancer).

Results: Histology showed a total of 46 discrete $\mathrm{G} \geq 4$ lesions among our 32 patients; 26 were G4 fused small glands (G4f), 13 were G4 cribriform glands (G4c), and 6 were G5. Quantitative PiCT maps of epithelium were most predictive of cancer location. Both PiCT and PI-RADS correctly found $10 \mathrm{G} 4 \mathrm{f}, 8 \mathrm{G} 4 \mathrm{c}$, and $5 \mathrm{G} 5$ lesions. PI-RADS alone located another $2 \mathrm{G} 4 \mathrm{f}$ lesions, and PiCT alone found an additional $11 \mathrm{G} 4 \mathrm{f}$ and 3 G4c lesions. Both missed $3 \mathrm{G} 4 \mathrm{f}, 2 \mathrm{G} 4 \mathrm{c}$, and 1 G5 lesion.

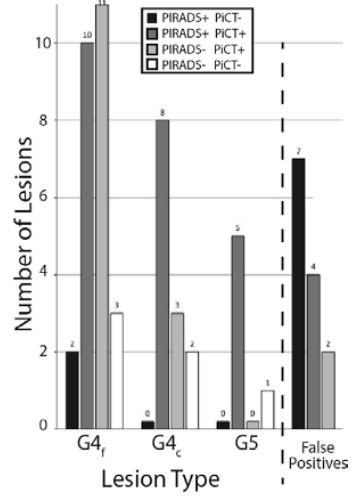

There were 7 false positives detected by PI-RADS alone, 4 by both methods, and 2 by PiCT alone.

Conclusions: We generated PiCT maps of histologic features based on MR imaging. Though overlap exists between PI-RADS 4+ lesions and PiCT maps, PiCT maps have superior true positive rate and lower false positive rate than PI-RADS reads.

\section{SMART NANOMOTORS : TOWARDS In Vivo SENSING}

Bincy Jose, Sharon O'Toole, Robert Forster, John J O'Leary. Trinity College Dublin, Dublin, Ireland; Dublin City University, Dublin, Ireland; The Coombe Women \& Infants University Hospital, Dublin, Ireland.

Background: Sampling bodily fluids, such as blood, saliva or urine, provides only a limited snapshot of an individual's health at an instant in time. Ultimately, truly personalised medicine needs to be in vivo with continuous monitoring of a wide range of health indicators. In vivo "Nanobots", i.e., nanoscale robots, are used for the delivery of medicines to specific disease causing cells. A key requirement is chemotaxis, i.e., movement along a concentration gradient of a disease biomarker, but it remains a huge challenge with the current state-of-the-art being preferential movement towards a fuel supply, typically hydrogen peroxide, rather than a biomarker release site.

Design: To advance in vivo personalised medicine by developing nanomachines that can be deployed within a patient's body that are capable of autonomous movement towards a disease biomarker release site and delivering a drug payload. This project focusses on fabricating nanomachines capable of autonomously switching on and off their nanomotors in response to the presence of a biomarker and signalling that they have detected the biomarker. Regioselectively modified particles are uniquely placed to deliver this functionality since one physical region detects the disease biomarker while the second acts as a nanomotor to drive the particle in a particular direction. Results: As illustrated in the figure below, metal nanoparticles are functionalised on one side with molecular switches or gates that open in response to a biomarker target so as to expose a catalyst. The exposed catalyst will then generate peroxide that acts as the nanomotor fuel propelling the nanobot in the direction of the biomarker plume. Conclusions: Nanorockets capable of selectively detecting diseased cells were fabricated and selectively functionalized different regions of the rockets for autonomous movement and cancer cell recognition.
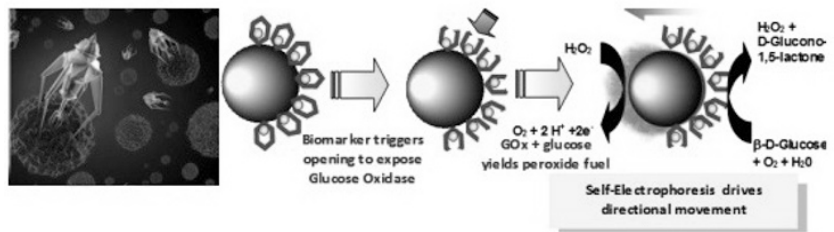

2133 Pathologic Quantification of Low Volume Discontinuous Metastatic Tumor Deposits (DMTD) in a Lymph Node (LN) - Analysis of AJCC $8^{\text {th }}$ Ed. Cancer Staging Manual and Survey of Expert Surgical Pathologists

Oleksandr N Kryvenko, Mahul B Amin, Henry D Appelman, Pedram Argani, John N Eble, Jonathan I Epstein, Linda Ferrell, John R Goldblum, Carmen Gomez-Fernandez, John C Kwok-Cheung, Merce Jorda, Ricardo VLloyd, Martin CMihm, Mehrdad Nadji, Victor E Reuter, Mark R Wick, Andrew E Rosenberg. University of Miami and Many Others, Miami, FL.

Background: Pathologic quantification of metastatic tumor in LNs is usually reported as the single largest size. Reporting the size of DMTD in a LN is not standardized. Design: We reviewed the AJCC $8^{\text {th }}$ Staging Manual and conducted a survey of 18 expert surgical pathologists who quantified the size of a LN metastasis composed of 4 closely situated carcinoma deposits. 


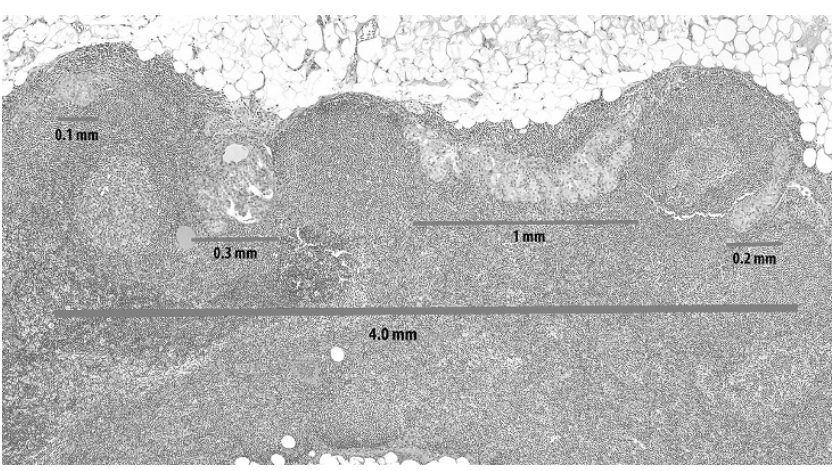

Results: AJCC $8^{\text {th }}$ Manual Chapter 1 defines isolated tumor cells ((ITC), $\leq 0.2 \mathrm{~mm}$ ) and micrometastasis $(>0.2-\leq 2 \mathrm{~mm})$. According to specific organ system criteria, these differences in size can be translated into negative and positive LNs, respectively. In 16 of 65 chapters the size of the metastasis is used for staging. Only melanoma and breast chapters describe that the size of the largest deposit should be recorded in a LN with DMTD. In H\&N chapter, micrometastasis is defined as $\leq 2 \mathrm{~mm}$ without a lower size threshold and ITC is considered positive for staging. Lower gastrointestinal tract ( 2 organ sites), breast, and female reproductive organs (6 organ sites) chapters define threshold of metastasis as $>0.2 \mathrm{~mm}$ but do not mention the approach to DMTD (except breast). Thyroid chapter suggests recording the maximum diameter of the metastasis and states there are no pertinent cutoff values for staging. The 18 expert surgical pathologists had diverse approaches and conclusions with significantly different clinical implications.

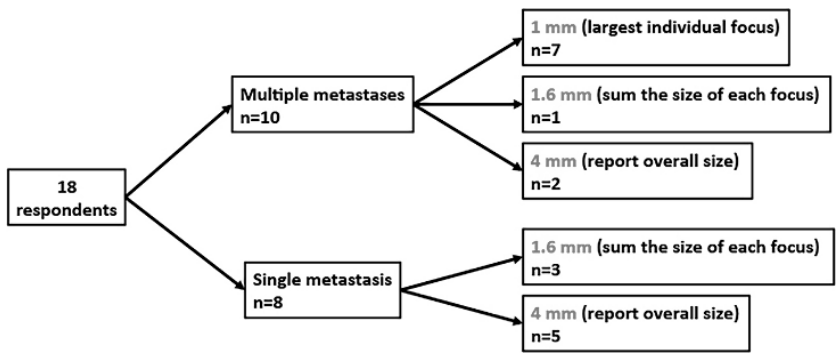

Conclusions: There are no consensus guidelines for the measurement of DMTD in LNs from many organs. Standardization even in the absence of medical evidence is an improvement over the current disparate state of affairs. We suggest therefore, that until supportive data are available, an objective approach is to document in the pathology report the presence of DMTD in a LN and describe the method for calculating the largest dimension of the metastasis.

2134 Short Duration Rapid Chilling of Mastectomy Specimens Improves Gross Examination for Breast Cancer and Optimally Preserves DNA for Molecular Studies

Asangi R Kumarapeli, William Bellamy, Ericka Olgaard, Nicole Massoll, Soheila Korourian. University of Arkansas for Medical Sciences, Little Rock, AR.

Background: A thorough gross examination of specimens for breast cancer requires the tissue to be very thinly sectioned, which is often quite difficult in large mastectomy specimens. We have implemented rapid chilling of mastectomies at $-80^{\circ} \mathrm{C}$ to facilitate thin sectioning prior to formalin fixation. The effects of this technique on ER, PR and Her2 biomarker testing have been previously evaluated. This study further examines the quality of tumor DNA after this process and its suitability for subsequent molecular genetic testing.

Design: Upon receipt in the laboratory, mastectomy specimens are weighed, measured, the margins inked and chilled at $-80^{\circ} \mathrm{C}$ for $20-30$ minutes. This process facilitates uniform sectioning of tissue at $4 \mathrm{~mm}$ intervals and aids in proper fixation and identification of small lesions. The integrity of chilled tissue for further testing was analyzed by three methods. First, demonstration of essentially $100 \%$ concordance between biomarker results performed on diagnostic core biopsies and the corresponding rapidly chilled mastectomies indicated retained protein integrity. Secondly, DNA from formalin-fixed and paraffin embedded (FFPE) mastectomy tissue with invasive carcinoma $(n=6)$ was extracted. The quantity and purity of DNA were assessed by spectrophotometry. DNA was amplified using AmpliTag Gold DNA polymerase kit (ABI, CA) with a Specimen Control Size Ladder master mix from the IGH gene rearrangement kit (InVivoScrive, CA). DNA fragment analysis was performed using Genetic Analyzer and GeneMapper (ABI). Lastly, all Oncotype DX (Genomic Health, CA) reports from 2011-2014 were reviewed to identify if any tissue sent from rapidly chilled specimens had documented assay interferences.

Results: The average 260/280 absorbance ratio was 1.82 (range 1.6 to 1.93 ), indicating suitable purity of DNA for subsequent studies. The concentration of recovered DNA ranged from 8.5 to $254 \mathrm{ng} / \mu \mathrm{L}$. By PCR, all samples produced amplified fragments of 300 to 400 base pair lengths that are expected of FFPE tissue. Out of 86 Oncotype DX reports analyzed, 43 were derived from rapidly chilled mastectomies. We did not find any tissue rejections or assay failures documented in these reports, indicating poor quality of tumor DNA.

Conclusions: Short duration rapid chilling of mastectomies improves detection of small lesions, optimally preserves DNA and does not interfere with molecular genetic studies. These findings complement our previous observations that this technique had no effect on routine biomarker testing.
2135 Pathologic Evaluation of a Novel Thermal Accelerant Used in Microwave Tumor Ablation

Kara A Lombardo, William KC Park, Aaron WP Maxwell, Edward G Walsh, Michael P Primmer, Scott A Collins, Damian E Dupuy, Shaolei Lu. Brown University Alpert Medical School, Providence, RI.

Background: The largest challenge in thermal ablation of liver tumors (hepatocellular carcinoma or cholangiocarcinoma) is a relatively high recurrence rate $(\sim 30 \%)$ for which incomplete ablation at the periphery is one likely reason. To overcome this challenge, we have developed a novel nontoxic liquid Thermal Accelerator (TA) that can be injected into areas unreachable by microwave antenna, solidify to gel at body temperature, and be heated by microwave energy to achieve an extended ablated area. We studied the pathologic effect of ablation performed with TA, as well as the ability of triphenyl tetrazolium chloride (TTC) stain to predict the ablation effect in in vivo animal studies. Design: TA performance was evaluated with a microwave ablation system using in vitro agarose phantom and in vivo porcine liver. Microwave power, TA dose, and TAto-tip distance were varied, and temperature readings compared with and without TA. The in vivo study involved liver ablation with and without TA in swine under general anesthesia. After ablation, livers were immediately harvested and serially sectioned for TTC staining. Unstained nonviable area in each section was digitally photographed and a digital-image analytic software (Image Pro Premier) was used to measure the ablated area and calculate total 3-dimentional volume. NADH stain on frozen tissue and H\&E examination were used to assist and validate the evaluation.

Results: Using the in vitro model, both rate and magnitude of increased temperature in the ablation zone were significantly greater with TA under all tested conditions $(\mathrm{p}<0.0001)$. In vivo studies, TTC stain demonstrated three distinct zones in the liver ablation site; central over-ablated zone, middle zone of coagulation necrosis and peripheral partially viable zone. Based on the staining, nonviable areas (central and middle zones) were calculated using Image Pro Premier. The overall nonviable areas were significantly increased with TA when either single or multiple antennae were used $(\mathrm{p}<0.01$ for both). NADH stain confirmed the presence of non-viable area and H\&E stain further characterized the morphologic features of the three zones.

Conclusions: We have developed a novel TA that can augment microwave energy, resulting in an enlarged ablated area in animal studies. Three distinct zones were identified in the ablation site, each with characteristic H\&E morphology. TTC proved to be an economic and accurate staining agent for visualizing viable liver tissue after ablation.

\section{$2136 \quad$ High Efficiency Multiplexing for Personalized Medicine}

Marco Manzoni, Carla R Scalia, Maddalena M Bolognesi, Giorgio Cattoretti. Università degli Studi di Milano-Bicocca, Monza, Italy; Ospedale San Gerardo, Monza, Italy. Background: Personalized oncological therapies are based on the genomic and transcriptomic analysis of the neoplastic tissue, obtained from nucleic acids extracted from the routinely fixed specimen used for the histological diagnosis. Pathology guidelines prioritize molecular analyses against an extensive IHC panel. However the biopsy specimen are often minute. Staining for multiple markers on the very same section (multiplexing) could overcome these limitations. Three approaches have been developed: directly labelled antibodies (Gerdes et al., 2013), sequential fluorochrometyramide deposition (Toth et al., 2007), ion-tagged, mass-spectrometry detected, isotope-labelled antibodies (nextGen IHC; Rimm D.L., 2014).

Design: We have been developing an high efficiency multiplexing (HEM) technology able to stain a single routine section with 20 markers or more. This technology is based on the use of indirect immunofluorescence and digital slide scanning, it can be used on any histological specimen and any disease, and allows a quantitative analysis. Results: HEM is based on indirect immunofluorescence with saturated solution of antibodies of different species (mouse, rabbit, goat) and different isotypes. This allows the composition of panels directed against relevant targets, detected three at a time (FITC, TRITC, Cy5+DAPI). A digital image of the whole section is acquired by a fast and high-definition digital slide scanner (Nanozoomer S60, Hamamatsu), before the section is stripped of the antibodies and re-stained. The process can be repeated in excess of 15 times. The method is free of cross-reactivity and steric hindrance (e.g. is possibile to show the simultaneous colocalization of CD3 and TCRbeta). Autofluorecence is eliminated through the method of dark pixel intensity with a dedicated 420/530 (exc/ em) filter set, allowing a significant improvement of the detection of specific signals.

\section{IsoData automatic thresholding in ImageJ}
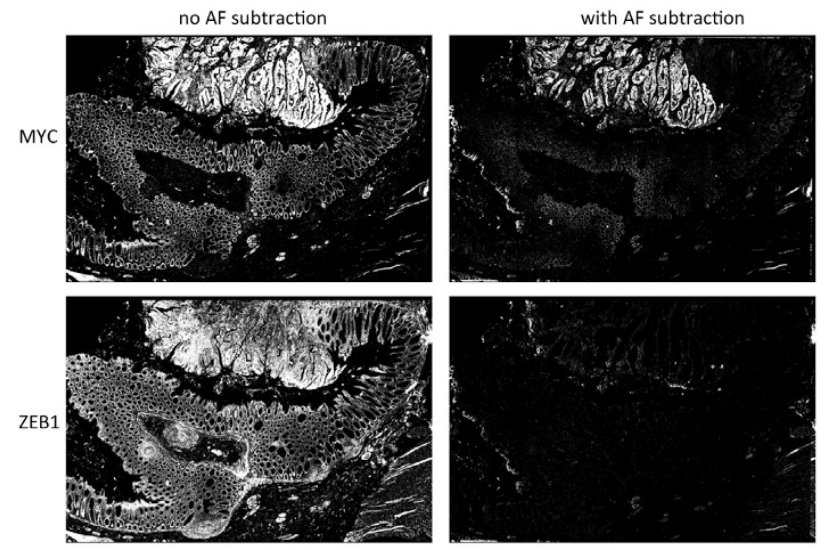
Sequential staining for Rb anti-MYC and Rb anti-Zeb1 of a colon cancer/normal gut section, thresholded with the same algorithm, with and w/o autofluorescence removal. Note the specific signal only on the right.

Conclusions: HEM is ought to become an high resolution and high throughput technology for personalized treatment in many areas.

2137 Clarification of Prostate Core Needle Biopsies with 2,2 Thiodiethanol Rapidly and Efficiently Prepares Tissue for 3D Light-Sheet Microscopy

Erin McCarty, Nicholas P Reder, Adam K Glaser, Ye Chen, Jonathan TC Liu, Lawrence True. University of Washington Medical Center, Seattle, WA; University of Washington, Seattle, WA.

Background: Emerging microscopy techniques, i.e. light-sheet microscopy (LSM), enable 3D visualization of intact tissue. However, the light scattering properties of intact tissue limit the depth of focus. One strategy to minimize light scattering in tissue is clarification via refractive index matching and/or delipidization. A wide range of clarification techniques have been developed to increase depth of focus, yet their complexity, time, and expense limit clinical feasibility. We describe a rapid, low cost, and effective clarification method using 2,2 ' thiodethanol (TDE), a refractive index matching solution.

Design: Phantom fluorescent beads suspended in agar were incubated with varying concentrations of TDE and allowed to clear for multiple times $(5,10,15,30,45$, and 60 minutes). After protocol optimization using the phantom beads, prostate core needle biopsies ( $\mathrm{N}=8$ ) were incubated with TDE, nuclear (DRAQ5), and cytoplasmic (eosin) fluorescent stains. Images of the cleared prostate biopsies were assessed for quality of staining and depth of focus. They were compared to gold standard H\&E slides derived from the same tissue. All microscopy was performed using a custom-built invertedstage LSM system.

Results: The optimal TDE concentration was $60 \% \mathrm{v} / \mathrm{v}$ with phosphate-buffered saline; clearing was complete by 15 minutes. Cleared prostate needle biopsies stained with DRAQ5 could be imaged with high resolution to a depth of 300-500 microns (Fig 1). Comparison with $\mathrm{H} \& \mathrm{E}$ slides showed indistinguishable features on low ( $4 \mathrm{x}$ objective field of view) and medium ( $\sim 10 \mathrm{x})$ magnifications. Interpretation of high magnification ( $\sim 0 \mathrm{x})$ LSM images was limited by the resolution constraints of the current LSM system. Conclusions: Our rapid, low cost TDE clarification protocol provides a clinically feasible method for slide-free, non-destructive 3D fluorescence microscopy of intact tissues. Further advances in microscopy and tissue clarification could enable rapid, high resolution visualization of entire needle core biopsies while preserving tissue for downstream molecular analyses.

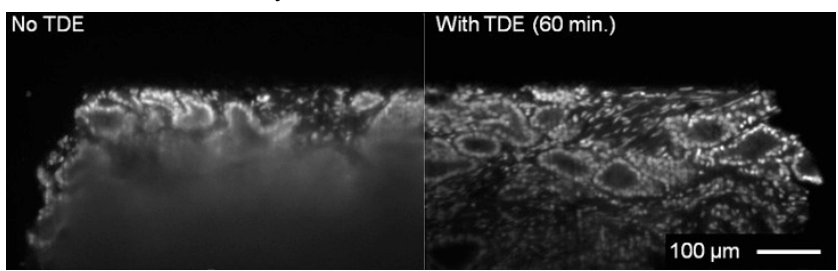

2138 Diagnostic Efficiency in Digital Pathology: A Comparison of Optical vs. Digital Assessment in $\mathbf{5 0 0}$ Surgical Pathology Cases

Anne Mills, Sarah E Gradecki, Christopher A Moskaluk, Bethany J Horton, Rebecca Blackwell, James W Mandell, Stacey E Mills, Helen P Cathro. University of Virginia, Charlottesville, VA.

Background: Prior work has shown that digital images and microscopic slides can be interpreted with comparable diagnostic accuracy. However, the interpretative time for digital images has scarcely been studied and concerns about efficiency remain a major barrier to adoption. We investigated the efficiency of digital pathology when compared to glass slide interpretation in the diagnosis of surgical pathology biopsy and resection specimens.

Design: Slides were pulled from 500 surgical pathology cases (100 gastrointestinal, 100 ovary, 100 endometrium, 100 bladder, 100 brain). The primary diagnosis and representative slides were validated by 2 pathologists (AM, CM). Diagnostic slides were scanned using the Philips IntelliSite Digital Pathology Solution slide scanner. Each case was assessed independently on digital and optical by 3 pathologists (HC, $\mathrm{JM}, \mathrm{SM}$ ), with $\mathrm{a} \geq 6$ week washout period between modalities. Reading pathologists recorded assessment times for each modality; digital times included time to load the case.Diagnostic accuracy was determined based on whether a rendered diagnosis differed significantly from the original diagnosis. Statistical analysis was performed to assess for differences in interpretative times across modalities.

Results: All 3 reading pathologists showed comparable diagnostic accuracy across optical and digital modalities $(\sim 4 \%$ disagreement rate for all 3 readers across both modalities). One reader showed no significant difference in digital vs. optical assessment times, 2 were slightly slower on digital (mean: 3 seconds and 13 seconds slower per case). Two readers showed a learning effect during the course of the study so that digital assessment times decreased over time. Organ site and specimen type did not significantly influence differences in interpretative times.

Conclusions: Digital images can be interpreted with robust efficiency relative to glass slides across a variety of organ systems and specimen types. Mean increase in assessment time across all three reviewers is 5 seconds/case, translating to $\sim 4-5$ minutes of increased work during a 50 case sign-out. This increased time can be minimized with practice and may be balanced by the improved ease of electronic chart access allowed by digital slide viewing.
2139 Molecular Lymph Node Analysis in Colorectal Carcinoma. Is HE Obsolete in the Molecular Era?

Carla Montironi, Iban Aldecoa, Natalia Rakislova, Josep Antoni Bombi, Nuria Chic, Joan Maurel, Francesc Balaguer, Maria Pellise, Antoni Castells, Miriam Cuatrecasas. Hospital Clinic, Barcelona, Spain.

Background: Lymph node staging $(\mathrm{pN})$ in colorectal carcinoma (CRC) is performed with $\mathrm{HE}$ and at least 12 lymph nodes (LNs) should be analyzed to state a reliable pN0 stage. Nevertheless, up to $25 \%$ of surgically treated stage II CRC patients may recur within 5 years of curative-intended surgery. Molecular LN analysis is more sensitive than HE, and makes it possible to detect the presence of tumor burden in pNO LNs, which has been associated with poor prognosis.

We aimed to quantify the amount of tumor cytokeratin 19 (CK19) mRNA present in LNs of colon cancer (CC) patients using RT-LAMP (Reverse Transcription Loop-mediated Isothermal Amplification), and to correlate the results with the patient's outcome.

Design: Analysis of 1449 LNs harvested from 102 surgically treated CC patients between June 2012 and December 2013 was performed with both, HE and RT-LAMP. We obtained the total tumor load (TTL) from each case, defined as the sum of CK19 mRNA copies $/ \mu \mathrm{L}$ from all positive $\mathrm{LNs}$ in a surgical specimen. Clinical and histological data, including the TTL, was correlated with disease recurrence data.

Results: A median of 13 LNs (IQR 10;18) were collected per patient. Univariate analysis showed that higher TTL was related to tumor location, tumor budding and presence of perineural and lymphatic invasion. Multivariate analysis showed a relationship with vascular invasion. Six patients $(6.3 \%)$ recurred at 3 years. A cutoff value for TTL $=7500$ CK19 mRNA copies/ $\mu \mathrm{L}$ was set as predictor of recurrence at 3 years; sensitivity $83.3 \%$ (95\% CI 43.6-97.0); specificity 84.9\% (95\% CI 72.9-92.1).The regression Cox model showed age and TTL as predictors of recurrence at 3 years $(\mathrm{p}=0.035) ; \mathrm{AUC}=0.722$.

Conclusions: The total tumor load makes it possible to quantify the amount of tumor burden present in LNs of CC patients, and could provide a more accurate LN pathological staging with potential prognostic and therapeutic implications.

\section{Comparison of PD-L1 Immunostain Concordance of SP263 and} SP142 Across Different Tumor Types

Manando Nakasaki, Oluwole Fadare, Sandip Patel, Donna Hansel. University of California at San Diego, La Jolla, CA.

Background: Immunotherapy using PD-L1 and PD-1 inhibitors have recently emerged as a unique and powerful method to treat a subset of cancer patients. Programmed cell death ligand-1 (PD-L1, B7 homolog 1, CD274) interacts with the PD-1 receptor on immune cells to impede the immune system recognition of cancer cells. Recently developed immunotherapy strategies that block this interaction can release immune inhibition and result in cancer cell targeting. PD-L1 immunostaining has been proposed as a possible indicator of enhanced therapy response, although the data has varied. We compared the ability of two Ventana-based antibodies to stain different cancer types and assessed their correlation in a clinical setting.

Design: We reviewed 87 prospectively analyzed specimens that were part of clinical analysis of PD-L1 immunostaining. We used 2 different antibodies (SP142, Spring Biosciences and SP263, Roche) on a Ventana Benchmark ULTRA platform on sequential tissue sections. Membranous and cytoplasmic immunoreactivity was characterized antibody-specific categorization. SP142 was assessed using cut-points of tumor cell (TC) or immune cell (IC) of TC0 or IC0 $(<1 \%$ cells), TC1 or IC1 $(>$ or $=1 \%$ to $<5 \%)$, TC2 $(>$ or $=5 \%$ to $<50 \%)$, IC2 $(>$ or $=5 \%$ to $<10 \%)$, TC3 $(>$ or $=50 \%)$ and IC3 $(>$ or $=10 \%$ ). Overall SP142 positivity was called if TC2 or IC2 criteria were reached. SP263 in TC and IC were additionally scored individually as negative ( $<25 \%$ of cells) or positive ( $>$ or $=25 \%$ cells), with either resulting in an overall positive assignment. Results: Using SP142, 19 cases were classified as overall positive (22\%). A positive interpretation resulted from TC scores of 2 or above in 10 cases (11\%)and IC scores of 2 or above in 11 cases (11\%), with 2 of these representing dual TC-IC positive staining. Using SP263, 12 cases were classified as positive (14\%), including 12 cases based on TC criteria and X cases based on IC criteria. Comparison between SP263 and SP142 showed a concordance rate of 0.78 and kappa 0.262 in designating a positive PD-L1 status. Conclusions: Our retrospective analysis of PDL-1 immunostaining showed that PD-L1 detection in cancer cells varied by antibody clone and the concordance between SP142 and SP263 was relatively poor. These results indicate that the clinical evaluation of the PD-L1 expression in patient tumor samples may vary by antibody and limit accurate detection of PD-L1 TC or IC expression. Additional refinement of criteria is needed to enhance predictability of immunotherapy response using this biomarker.

\section{Deep Learning Classifier to Predict Cardiac Failure from Whole-} Slide H\&E Images

Jeffrey Nirschl, Andrew Janowczyk, Eliot Peyster, Renee Frank, Kenneth Margulies, Michael Feldman, Anant Madabhushi. University of Pennsylvania, Philadelphia, PA; Case Western Reserve University, Cleveland, $\mathrm{OH}$.

Background: Cardiovascular diseases are the leading cause of death globally, and over 26 million people worldwide suffer from heart failure. When the cause of heart failure is unknown, endomyocardial biopsy (EMB), is the gold standard for evaluation and grading of heart disease. EMB is usually employed (a) after clinical symptoms develop or (b) as routine monitoring in heart transplant patients. The primary concern with the interpretation of EMB is relatively high inter-rater variability in the pathologic diagnosis (e.g., Cohen's kappa= .39 in grading rejection cases). In this work, we show that computational histomorphometric image analysis (CHIAl) of digitized images of stained sections from EMBs in conjunction with deep learning algorithms is able to predict heart failure. The foundation for CHIAI is a powerful machine learning approach termed deep learning (DL), which learns feature representations directly from the data to predict tissue-level pathology. 
Design: Our approach (see Figure 1) was evaluated on H\&E stained digitized wholeslide images (WSI) obtained from 209 patients with clinical heart failure (receiving heart transplants, $\mathrm{N}=96$ ) or without known history of heart failure (potential heart donors, $\mathrm{N}=113$ ), determined by clinical history and cardiac ultrasound. 104 patients were randomly identified from the set of 209 and designated for training (and used for 3-fold cross-validation), and 105 patients were held out as an independent test set. From each WSI, 11 non-overlapping regions of interest of $250 \times 250$ pixels were randomly cropped. About 278,000 patches of $64 \times 64$ pixels were extracted from this set to train a DL model consisting of 6 sets of convolutional, batch normalization, and rectified linear layers, followed by a fully-connected layer culminating in a softmax classification layer. Patient-level predictions are derived by majority vote from the 11 image level predictions.

\section{Figure 1}

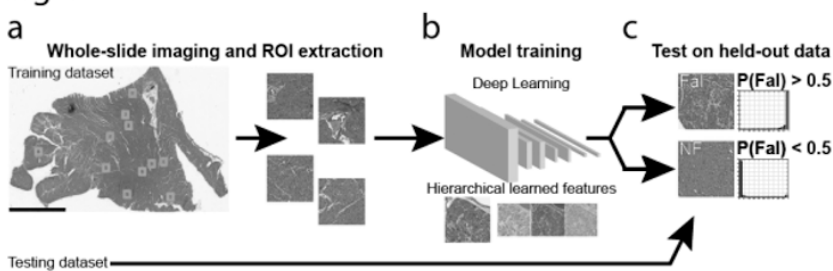

Schematic overview of digital pathology workflow to predict heart failure. (a) Patients were divided into a training and test dataset. WSI were scanned and regions of interest (ROI) extracted for image analysis. All ROIs from the same patient were given the same label, which was determined by whether the patient had clinical or pathological evidence of heart disease. (b) Three-fold cross validation was used to train heart failure classifiers for our deep learning model. (c) Trained models were evaluated at the image and patient-level on a held-out test dataset.

Results: Our approach was validated at the patient level, yielding a mean accuracy of $96.2 \% \pm 2 \%$ during 3 -fold validation on the training set and $96.2 \% \pm 1 \%$ on the independently held-out test set.

Conclusions: We presented a DL scheme which accurately predicts, from features mined from digitized images of EMBs, which patients have heart failure.

2142 Optimal Conditions of FFPE Samples for Extraction and Molecular Analysis of DNA for Atomic Bomb Survivors

Takashi Ohnoda, Katsuya Matsuda, Yuko Akazawa, Nozomi Ueki, Yuka Yamaguchi, Shiro Miura, Akira Kinoshita, Koh-ichiro Yoshiura, Masahiro Nakashima. Nagasak University School of Medicine, Nagasaki, Japan; Atomic Bomb Disease Institute, Nagasaki University, Nagasaki, Japan; Nagasaki University Hospital, Nagasaki, Japan; Atomic Bomb Disease Institute, Nagasaki, Nagasaki, Japan.

Background: Radiation exposure has long-term effects that contribute to carcinogenesis. Atomic bombing (A-bombing) survivors are the only available cohort in which these effects can be examined. Thus, efficient sample collection, storage, and comprehensive genome studies are needed to study radiation-induced effects in this cohort. Our institution has obtained numerous cancer tissue samples of A-bombing survivors, and these have been stored as FFPE samples for decades. However, poor fixation conditions promote DNA degradation. We assessed the fixation conditions used in preparing these FFPE samples and evaluated their DNA integrity.

Design: Colorectal cancer FFPE samples were obtained from subjects $(n=5)$ who had undergone surgery in 2014-15. Tissues were cut into 10 pieces and fixed in non-buffered (NBF) or buffered formalin (BF) for 1-30 d. Fresh-frozen tissues from the same samples were used as controls. DNA was extracted and its quality was analyzed.

Results: Ethidium bromide-stained agarose gel showed fixation time-dependent fragmentation of NBF-DNA. The dsDNA/total DNA ratio was 35\% in NBF-DNA and $56 \%$ in BF-DNA; further, in NBF-DNA, this ratio decreased as fixation time increased. In the PCR analysis, the $>200$-bp amplificability decreased remarkably after $7 \mathrm{~d}$ of NBF fixation, while BF-DNA showed good template activity even after $30 \mathrm{~d}$ of fixation. In the ddPCR analysis, the copy numbers of PTEN and vimentin decreased after $3 \mathrm{~d}$ of NBF fixation and $7 \mathrm{~d}$ of BF fixation. The $P T E N /$ vimentin copy number ratio was $0.6-0.4$ in NBF-DNA and 0.8-0.7 in BF-DNA; in contrast, the ratio was $\sim 1.0$ in control DNA. Further, in NBF-DNA, this ratio decreased as fixation time increased. In the FISH analysis, the PTEN and CEP10 signals decreased from day 3 of NBF fixation and day 7 of $\mathrm{BF}$ fixation. The PTEN/CEP10 signal ratio decreased markedly in long-term formalin-fixed samples.

Conclusions: Short-term BF-fixed samples were useful for various analyses, while longterm NBF-fixed samples degraded rapidly. Cancer tissues from A-bombing survivors should be stored as fresh-frozen tissue and BF-fixed FFPE samples should be prepared within $3 \mathrm{~d}$ as a secondary option.

2143 TTF-1 and Napsin A Expression in Lung AdenocarcinomaEvaluation by Double Stain Immunohistochemistry and RNA ISH Assay Claudia Ormenisan Gherasim, Diane Lawson, Wanhong Jiang, Momin T Siddiqui, Cynthia Cohen. Emory University, Atlanta, GA.

Background: Non-small cell lung cancer (NSCLC) accounts for $80 \%$ of lung cancers. Lung adenocarcinoma (ADC) is one of the main types of NSCLC. Recent studies show that patients with high expression levels of TTF-1 and Napsin A, and high co-expression levels of TTF-1/Napsin A, have better survival rates than those with low levels of expression. Therapy for NSCLC is currently determined by histologic subtype and the presence or absence of actionable mutations. Accurate subclassification is therefore essential for appropriate selection of cases for molecular studies and guiding treatment. The aim of this study was to investigate the expression of TTF-1 and Napsin A in lung ADC, comparing two different technologies: immunohistochemistry (IHC) versus RNA in situ hybridization (RISH)
Design: We selected 20 cases of adenocarcinoma of the lung and 4 samples of metastatic colon adenocarcinoma. Evaluation of TTF1 and Napsin A expression was performed by double stain IHC, TTF-1/Napsin A (brown nuclear TTF1 [Dako, 1/80] and red cytoplasmic Napsin A [Leica 1/400]) and RISH. RNA-ISH was performed using RNAscope (Leica Biosystems, Buffalo Grove, IL) with the Bond III immunostainer (Leica), and probes Hs-NKX 2.1 and Hs-Napsa (Advanced Cell Diagnostics, Newark, CA). Specific staining signals were identified as brown, punctate dots present in the cytoplasm and/or nucleus. Both methods were scored in tumor cells and quantified using combined intensity and proportion scores.

Results: Nineteen of twenty lung ADC (95\%) were positive for both TTF1/Napsin A IHC double stain. When evaluated by RNAscope, nineteen were positive for TTF1 $(95 \%)$ and eighteen for Napsin A. (90\%). None of the metastatic colon ADC stained for TTF 1 or Napsin A by either method.

\begin{tabular}{|l|l|l|l|}
\hline Method & TTF-1/Napsin-A IHC & TTF-1 RISH & Napsin-A RISH \\
\hline Sensitivity & $95 \%$ & $95 \%$ & $90 \%$ \\
\hline Specificity & $100 \%$ & $100 \%$ & $100 \%$ \\
\hline PPV & $100 \%$ & $100 \%$ & $100 \%$ \\
\hline NPV & $80 \%$ & $80 \%$ & $66 \%$ \\
\hline
\end{tabular}

Conclusions: When comparing double staining by IHC versus RNA ISH of TTF1 and Napsin-A, results are almost similar. IHC proves to be a cheaper and faster method to perform. IHC double stain requires only one slide, with more tissue saved for molecular studies.

\section{Performance Evaluation of Copan's Elution Swab Collection and Transport System for Preservation and Recovery of Mycobacteria and Fungi}

Dennise E Otero, Lawrence Esper, Lorraine Sykes, Suresh B Selvaraju. MetroHealth Medical Center, Cleveland, OH.

Background: The ESwab consists of a flocked nylon swab for sample collection and liquid Amies transport medium. ESwab is considered better for recovery of both aerobic and anaerobic bacteria compared to traditional swabs. However, there are no published data on ESwab efficiency in recovering mycobacteria and fungi. The objective of this study was to evaluate the ESwab for preservation and recovery of mycobacteria and fungi.

Design: Mycobacteria, yeasts and molds (M. avium, M. kansasii, M. chelonae, M. abscesses, M. gordonae, C. albicans, C. glabrata, C. krusei, Cryptococcus neoformans, A. fumigatus, Mucor sp., Fusarium sp., and Trichophyton sp.) were tested. The standardized initial inoculum equal to $0.5 \mathrm{McFarland}$ Standard (MFS; $\sim 1 \times 10^{8} \mathrm{CFU} /$ $\mathrm{ml}$ ) was prepared and subsequently, two 100 -fold serial dilutions (D1 and D2) were made. The ESwabs were inoculated with these three dilutions in duplicates. Inoculated swabs were processed per laboratory practice and plated at $0 \mathrm{~h}, 24 \mathrm{~h}$ and $48 \mathrm{~h}$ after incubating at room temperature. The colony forming units (CFU) were counted and the difference in recovery rate was calculated.

Results: In general, good preservation of viability and recovery of all test organisms at higher inoculum level of $0.5 \mathrm{MFS}$ was observed after $24 \mathrm{~h}$ and $48 \mathrm{~h}$ incubation. At D1 concentration after $24 \mathrm{~h}$ of incubation, all mycobacteria showed lower recovery rate of 0.65 folds except for M. gordonae, which showed 1.3 folds increase. However, at lower level of initial inoculum (D2), a reduced recovery of mycobacteria in the range of $0.3-0.9$ fold was seen after $24 \mathrm{~h}$ or $48 \mathrm{~h}$. Overall, both yeasts and molds recovered very well after 24 and 48 hours of incubation in both D1 and D2 concentrations. All yeasts in both D1 and D2 concentrations, showed 3 - 257 folds increase after $24 \mathrm{~h}$ or 48 hours. Similarly, molds also showed improved recovery in the range of $1.3-23.6$ folds after 48 hours.

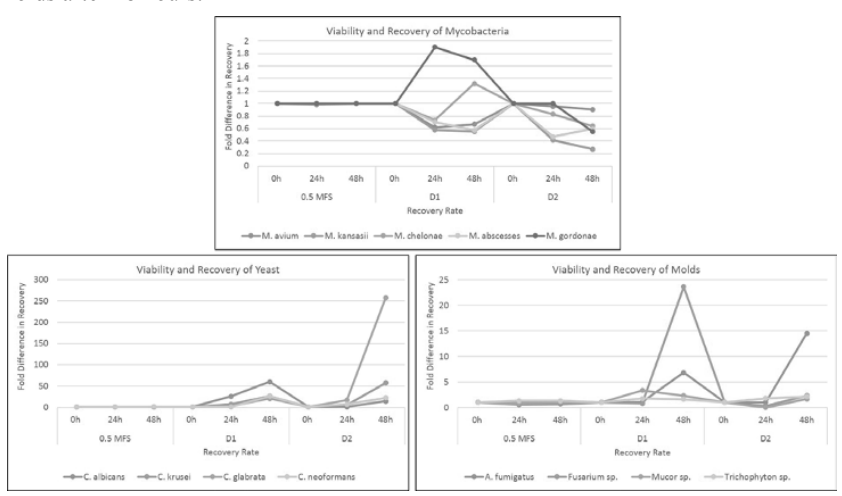

Conclusions: The ESwab showed acceptable performance in preserving and recovery of mycobacteria and fungi. However, immediate submission of ESwab specimens are recommended for mycobacteria. The ESwab can be used for routine mycobacterial and fungal culture when the preferred tissue or aspirate specimens are not available. 
$2145 \quad$ Ability of Desorption Electrospray Ionization (DESI) Mass Spectrometry Imaging (MSI) to Discriminate Between Tumors and NonNeoplastic Tissue in Resection Specimens from Multiple Organs

Kevin Yi Mi Ren, Martin Kaufmann, Nicole Morse, Amanda Shuo Xu, John Rudan, David Berman, Sonal Varma. Queen's University, Kingston, ON, Canada.

Background: Intraoperative diagnosis remains a challenging aspect of pathology practice that would benefit from ancillary tests capable of supplementing conventional frozen sections. DESI MSI is an emerging technique capable of tissue characterization based on metabolite profiling. It can be applied directly to unstained frozen section slides to create tissue images based on the relative abundance of metabolites that topographically match the histology sections while maitaining tissue integrity and spatial orientation. We present a proof-of-concept study to demonstrate the ability of DESI MSI to identify and delineate tumor from adjacent non-neoplastic tissue in breast, liver and kidney resections.

Design: Fresh tumor samples with adjacent non-neoplastic tissue were obtained from breast $(\mathrm{n}=2)$, liver $(\mathrm{n}=3)$ and kidney $(\mathrm{n}=3)$ specimens containing grossly identifiable tumors that were histologically confirmed as invasive ductal, metastatic colorectal, and clear cell renal cell carcinomas, respectively. Unstained frozen section slides were subjected to DESI MSI analysis, and subsequently stained by hematoxylin and eosin (H\&E) for histologic correlation.

Results: A range of metabolites were found to accurately differentiate malignant from adjacent non-neoplastic regions within the same section. Metabolites with different mass-to-charge ratios $(\mathrm{m} / \mathrm{z})$ show distinct relative abundance in tumor $(\mathrm{m} / \mathrm{z}=309,331$, $788)$ and non-neoplastic tissue $(m / z=215,277,790)$ respectively in all tissue types. DESI MSI based on these metabolites was able to consistently delineate tumor from adjacent non-neoplastic tissue regardless of the tumor type or organ studied.

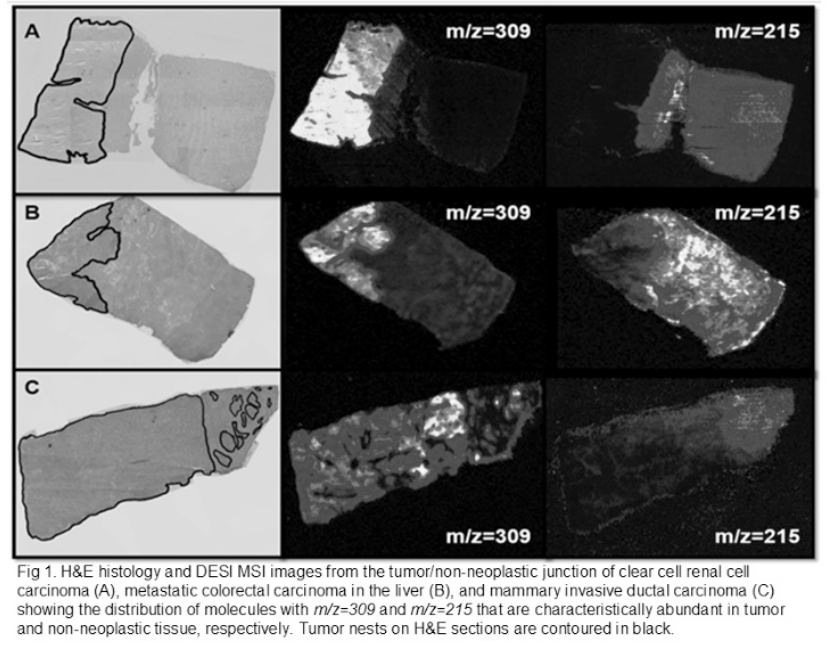

Conclusions: Our findings demonstrate that DESI MSI can be used to supplement histology in identifying and delineating tumor from non-neoplastic tissue in multiple solid organs, and may offer the potential to improve intraoperative diagnosis and surgical decision making. Additionally, the metabolic signatures obtained from this study would help build a metabolic library to guide in vivo tumor assessment techniques like 'iKnife' that our group is currently investigating.

2146 Massively Parallel Sequencing Approach for the Detection of XP11 Translocation in Renal Cell Carcinoma

Omid Rouhi, Tong Yang, Jordan Magee Owens, Heather H Jones, Wanhong Jiang. Momin T Siddiqui, Charles E Hill, Cynthia Cohen. Emory University, Atlanta, GA. Background: The Xp11 translocation Renal Cell Carcinomas (RCC) are characterized by gene fusions involving TFE3. The result is a TFE3 transcription factor gene fusion with one of multiple reported genes including ASPL (ASPS), PRCC, SFPQ (PSF), NonO (p54), and CLTC. Break-apart TFE3 fluorescence in situ hybridization (FISH) on formalin-fixed paraffin-embedded (FFPE) tissue sections is currently the gold standard for identification of TFE3 rearrangements. Recently, next-generation sequencing (NGS) techniques at the transcriptome level (RNA-Seq) have been used to verify known and discover novel transcribed gene fusions.

Design: RNA was extracted from FFPE tissue of 16 RCC cases. The NGS libraries for targeted capture of mRNAs produced from fusion genes were prepared. The cDNA library was then loaded onto an Illumina platform sequencer followed by data analysis. Results: All FISH positive samples showed strong evidence of the TFE3 fusion. The NGS assay was able to show strong evidence of TFE3 fusion in one FISH negative case, and the absence of the fusion in 4 IHC positive cases. Interestingly, we detected a TFE3-LINC01010 fusion in one case with IHC positive and FISH negative results. The LINC01010, long intergenic non-protein coding RNA1010 is a novel fusion partner gene of TFE3.

\begin{tabular}{|c|c|c|c|c|}
\hline$\#$ & IHC & FISH & NGS & Partner Gene \\
\hline 1 & + & + & + & SFPQ \\
\hline 2 & - & + & + & PRCC \\
\hline 3 & + & + & + & PRCC \\
\hline 4 & + & - & + & LINC01010 \\
\hline 5 & - & - & - & \\
\hline 6 & + & - & - & \\
\hline 7 & - & - & - & \\
\hline 8 & + & - & - & \\
\hline 9 & - & - & - & \\
\hline 10 & + & & - & \\
\hline 11 & + & & + & \\
\hline 12 & + & & - & \\
\hline 13 & - & & - & \\
\hline 14 & - & & - & \\
\hline 14 & + & & - & \\
\hline 16 & + & & + & \\
\hline & & & & \\
\hline
\end{tabular}

Conclusions: The NGS based fusion gene assay is a robust technique to detect gene rearrangements from FFPE material. This assay is able to identify the novel fusion gene products which may be the target of future therapy. Furthermore, the interpretation of NGS results are less subjective than those of FISH and IHC tests.

2147 Dual-Color, Break-Apart FISH Assay on Paraffin-Embedded Tissues as an Adjunct to Diagnosis of Small Round Cell Sarcomas with CIC Translocation

Christian Salib, Daniel L Van Dyke, Patricia Adem, John T Fallon, Chitra Kumar, Minghao Zhong. Westchester Medical Center/ New York Medical College, Valhalla, NY; Mayo Clinic, Rochester, MN.

Background: Recently, CIC rearrangement was identified in EWS rearrangement negative Ewing-like sarcomas. These tumors are a clinically aggressive subgroup of undifferentiated round cell sarcomas demonstrating most commonly a CIC-DUX4 translocation and presenting over a wide range of patient age groups, most commonly in young adults. Nevertheless, their clinical presentations are exceedingly rare. To the best of our knowledge, there are no publications in current literature regarding the detection of CIC translocations via break-apart FISH assays.

Design: 3 sarcoma cases were collected and proven negative for WT1-EWR translocation using FISH. One case showed the typical $\mathrm{t}(4 ; 19)$, one case showed a novel $\mathrm{t}(1 ; 19)$ and the third case showed complex structural abnormality of chromosome 19q13. 2 custom-labeled, dual-color break-apart FISH probes flanking the CIC gene on chromosome 19 were generated (centromeric red, telomeric green). FISH assay was performed on metaphase of normal blood cells, neoplastic cells and adjacent non-neoplastic cells.

Results: Normal metaphase of blood cells showed both red and green signals on chromosome 19q13. Conversely, break-apart signals were observed in tumor cells of all 3 cases but not in adjacent non-neoplastic cells. These signals were consistent with the known CIC gene translocations.

Conclusions: This study demonstrated that dual-color break-apart FISH assay can serve as an adjunct technique for the diagnosis of tumors with CIC gene rearrangement on paraffin embedded tissue, including other novel CIC-related translocations.

2148 An Exploratory Study Employing Hepatocyte Nuclear FOXM1 Immunoexpression by Vectra ${ }^{\circledR}$ Automated Multispectral Imaging System: Inching Towards Quantitative Pathology as a Diagnostic Tool

Christine J Salibay, Yi-Fan Chen, Ryan Deaton, Luis Manon, Janais Peace, Michael Walsh, Pradip Raychaudhuri, Frederick Behm, Grace Guzman. University of Illinois Hospital \& Health Sciences System, Chicago, IL; Center for Clinical and Translational Science, Chicago, IL.

Background: Proto-oncogene FOXM1 is known to be upregulated in many human cancers including HCC. It is reported to be absent to minimal in nuclei of normal liver cells and upregulated in HCC. We investigated hepatocyte FOXM1 expression employing a quantitative pathology tool, Vectra ${ }^{\circledR}$ automated multispectral imaging system, in various stages of disease including normal, cirrhosis, dysplasia and HCC, and its correlation with gender, race, and other co-morbidities.

Design: FOXM1 immunohistochemistry was performed on a liver cancer progression tissue array consisting of paired liver tissue cores representing 6 normal, 141 cirrhosis, 211 dysplasia, and 79 HCCs tissues for 212 subjects. Nuclear size and stain, and cytoplasmic stain were evaluated using Vectra ${ }^{\circledR}$. Data was compared using generalized mixed models with a random intercept to take into account subject dependence. Subjects were placed in four quartiles of each measurement and each group was assessed on chances of having HCC in odds:ratio (OR). For subjects with demographics data $(\mathrm{n}=142)$, we fitted additional models adjusting for those covariates.

Results: Highest nuclear staining by FOXM1 was seen in HCC versus cirrhosis and dysplasia [Figure 1]. 


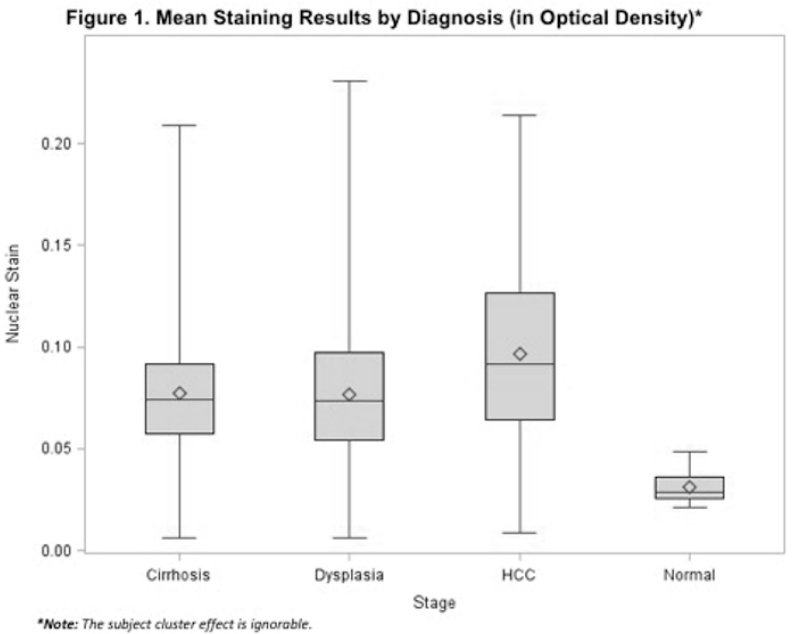

Using nuclear stain, the first (OR $0.263,95 \%$ CI 0.122 to 0.564 ), second (OR 0.375 , $95 \% \mathrm{CI} 0.182$ to 0.770 ), and third (OR $0.346,95 \%$ CI 0.167 to 0.718 ) quartiles were less likely to have HCC than the fourth with the highest increased FOXM1 expression. In linear mixed models, increase in nuclear size and stain were directly related to HCC. From analysis of subgroups, African American race $(\mathrm{p}=0.004)$ and DM II $(\mathrm{p}=0.050)$ were predictive of nuclear size and stain, respectively.

Conclusions: By employing Vectra ${ }^{\circledR}$ imaging, hepatocyte nuclear expression of FOXM1 mean is upregulated in HCC in comparison to other disease states. Furthermore, additional correlations using nuclear stain and size can be elucidated. Although none of these parameters by themselves are highly sensitive and specific, the advent of quantitative pathology shows promise to aid in accurate HCC diagnosis.

\section{Establishing a Pyrosequencing Assay for Determination of MLH1 Methylation Status}

Jan Seitz, Evgeny Moskalev, Florian Haller, Gerhard Seitz, Arndt Hartmann. FriedrichAlexander University, Erlangen, Germany; Klinikum Bamberg, Bamberg, Germany. Background: Deficiency of the DNA mismatch repair (MMR) system is characteristic for a group of colorectal cancers (CRC), e.g. microsatellite instable (MSI) CRCs. This deficiency is either caused by germline mutations or can occur sporadically via aberrant $M L H I$ methylation with ensuing loss of the protein. In CRC, BRAF mutation analysis is currently used as a surrogate parameter for aberrant $M L H 1$ methylation, as the presence of the $B R A F \mathrm{~V} 600 \mathrm{E}$ mutation is significantly correlated with $M L H I$ methylation. In endometrial cancer (EC) the frequency of the $B R A F \mathrm{~V} 600 \mathrm{E}$ is so low that a similar correlation is unlikely. For the current study, the aim was to develop a pyrosequencing assay for quantification of $M L H 1$ methylation in DNA from formalinfixed and paraffin-embedded (FFPE) tumor tissue, and to evaluate thresholds for aberrant $M L H 1$ methylation in colorectal cancer and endometrial cancer.

Design: $B R A F$ mutation analysis was performed using a self-designed pyrosequencing assay, tested using cell lines (HCT-116, HT-29) with known BRAF mutation status. The $M L H 1$ promoter region was analysed in silico for the presence of $\mathrm{CpG}$ sites and compared to published data on aberrant methylation status in 52 FFPE CRC patient samples. Accordingly a pyrosequencing assay interrogating the methylation level at 5 individual $\mathrm{CpG}$ sites was designed and tested using DNA samples with known methylation percentage. Additionally, FFPE samples of endometrial cancer were analysed for $M L H I$ methylation status and $B R A F \mathrm{~V} 600 \mathrm{E}$ mutation status.

Results: By using CRC samples containing the V600E mutation as positive controls and by comparison with immunohistochemistry, mean $\mathrm{MLHI}$ methylation levels of $>20 \%$ were regarded as methylated. 14/14 MSI CRCs likely to be germline mutated cases were negative for $M L H 1$ methylation and 11/14 were $B R A F$ wildtype. 20/23 likely to be sporadic MSI CRCs showed $M L H I$ methylation and 18/23 showed $B R A F$ mutation. $15 / 15$ MSS CRCs were negative for $M L H 1$ methylation as well as for $B R A F$ mutation. However while some patients in the EC group showed $M L H 1$ methylation there were none $B R A F$ mutations detected.

Conclusions: Depending on $B R A F$ mutation status alone when examining CRCs would have led to a lower sensitivity in this analysis. The combined results of $B R A F$ mutation and $M L H I$ methylation show the best outcome. Direct measurement of $M L H I$ methylation levels may be superior to BRAF mutation analysis in MSI-H cancers with a low frequency of $B R A F$ mutations (e.g. EC), since no correlation between $B R A F$ mutations and $M L H 1$ methylation could be observed for ECs.

2150 Characterizing FoxM1 Immunoexpression of Clear Cell and Papillary Renal Cell Carcinoma Employing Vectra Automated Multispectral Imaging System

Snehal Sonawane, Ryan Deaton, Alexandru Susma, Shankar Sonawane, Frederick Behm, Grace Guzman, Suman Setty. University of Illinois Hospital \& Health Sciences System, Chicago, IL; Vizient Inc, Chicago, IL.

Background: Fork head box M1 (FoxM1), a human proto-oncogene transcription factor governing cell proliferation, is known to be up regulated in many human cancers including renal cell carcinoma. Our aims were to characterize FoxM1 immunohistochemical expression in clear cell renal cell carcinoma (ccRCC) and papillary renal cell carcinoma (pRCC) using the Vectra Automated Multispectral Imaging System (VAMIS), and to determine distinguishing features including nuclear size, Fuhrman grade, and tumor stage.

Design: Fox M1 immunohistochemistry (IHC) was performed on a tissue microarray (TMA) with 204 cores sampled from 91 patients $(69 \mathrm{ccRCC}$ and $22 \mathrm{pRCC}$ in at least duplicate cores) by standard methods. The histopathological diagnosis and tumor grade of each core was confirmed independently by 3 observers using published criteria. The TMA was scanned and analyzed employing the VAMIS including nuclear size (positive pixels counts/square millimeter(ppc/mm2)), FoxM1 nuclear and cytoplasmic staining (optical density OD). Mean nuclear size, FoxM1 nuclear and cytoplasmic staining intensity were calculated using SQL Query analyzer. The relationship of FoxM1 staining features with Fuhrman grade, and tumor stage was also assessed using Microsoft excel, 2014

Results: Staining features of FoxM1 are summarized in Figure 1 and Figure 2.

\begin{tabular}{|c|c|c|c|c|c|c|}
\hline \multicolumn{7}{|c|}{ Averaged size and staining $\mathrm{re}$} \\
\hline & \multicolumn{3}{|c|}{ Clear Cell Renal Cell Carcinoma (CRCC) } & \multicolumn{3}{|c|}{ Papillary Renal Cell Carcinoma (pRCC) } \\
\hline $\begin{array}{c}\text { Fuhrman } \\
\text { Grade }\end{array}$ & $\begin{array}{c}\text { Nuclear Stain } \\
\text { Mean (OD) }\end{array}$ & $\begin{array}{c}\text { Cytoplasmic Stain } \\
\text { Mean (OD) }\end{array}$ & $\begin{array}{c}\text { Nuclear Size } \\
\text { Mean }(\mathrm{ppc} / \mathrm{mm} 2)\end{array}$ & $\begin{array}{l}\text { Nuclear } \\
\text { Stain Mean } \\
\text { (OD) }\end{array}$ & $\begin{array}{l}\text { Cytoplasmic } \\
\text { Stain Mean } \\
\text { (OD) }\end{array}$ & $\begin{array}{c}\text { Nuclear Size } \\
\text { Mean (ppc/mm2) }\end{array}$ \\
\hline 1 & 0.192617194 & 0.121401451 & 110.6208785 & 0.206002154 & 0.12706519 & 113.820803 \\
\hline 2 & 0.258849658 & 124481 & 2.4327493 & 0.275391329 & 0.205977951 & 109.5704356 \\
\hline 3 & 0.282765112 & 0.201748022 & 9.1629765 & 0.346459389 & 0.249850848 & 112.2228745 \\
\hline 4 & 0.259727533 & 0.173101327 & 113.7853724 & 0.199246438 & \begin{tabular}{l|l|}
8 & 0.193460701 \\
\end{tabular} & 107.1266439 \\
\hline \multicolumn{7}{|c|}{ Averaged size and staining results by Diagnosis and Tumor Stage } \\
\hline & \multicolumn{3}{|c|}{ Clear Cell Renal Cell Carcinoma (CRCC) } & \multicolumn{3}{|c|}{ Papillary Renal Cell Carcinoma (PRCC) } \\
\hline $\begin{array}{l}\text { Tumor } \\
\text { Stage }\end{array}$ & $\begin{array}{l}\text { Nuclear Stain } \\
\text { Mean }\end{array}$ & $\begin{array}{l}\text { Cytoplasmic Stain } \\
\text { Mean (OD) }\end{array}$ & $\begin{array}{c}\text { Nuclear Size } \\
\text { Mean (ppc/mm2) }\end{array}$ & $\begin{array}{c}\text { Nuclear Stain } \\
\text { Mean }(O D)\end{array}$ & \begin{tabular}{|c} 
Cytoplasmic \\
Stain Mean (OD)
\end{tabular} & $\begin{array}{c}\text { Nuclear Size } \\
\text { Mean (ppc/mm2) }\end{array}$ \\
\hline 1 & 0.253752089 & 0.174711078 & 111.9392411 & 0.261628924 & 0.18968483 & 107.4816145 \\
\hline II & & & & 0.408177687 & 23 & 109.5000235 \\
\hline IIII & 0.240666648 & 7638088 & 111.9999241 & 0.295122771 & 0.204554256 & 114.0452113 \\
\hline Iv & 0.308101881 & 0.207627876 & 111.7644333 & 0.199246438 & 0.123366755 & 120.0042216 \\
\hline
\end{tabular}

Conclusions: This study shows significant upregulation of FoxM1 with advancing Fuhraman grade 1 to 4 in ccRCC ( $<0.03$ for all). While there was a significant difference between grade 1 and grade $4(\mathrm{P}=0.03)$ in ccRCC groups, we postulate that the drop in FoxM1 features from grade 3 to 4 could be attributed to enhanced necrosis, and decrease detectable viable cells in higher grade tumors. The pRCC shows a trend towards increase with advancing tumor stage and grade but did not achieve statistical significance. There was a trend towards heightened FoxM1 expression in $\mathrm{pRCC}$ versus $\operatorname{ccRCC}(\mathrm{p}=0.03$ for cytoplasmic staining, and $\mathrm{p}=0.3$ for nuclear staining). The mean nuclear size showed a minor trend of increase in size as Fuhrman grade and tumar stage progresses and did not achieve statistical significance.

2151 Next-Generation Sequencing: Clinical Implementation in the Routine Diagnosis of Translocation-Associated Bone and Soft Tissue Tumors

David Swanson, Sharon Crafter, Andrew Wong, Evangeline Agro, George Charames, Rita A Kandel, Brendan C Dickson. Mount Sinai Hospital, Toronto, ON, Canada; University of Toronto, Toronto, ON, Canada.

Background: Next-generation sequencing (NGS) is a powerful technique with unprecedented diagnostic and research applications. Many tumors of bone and soft tissue - in addition to hematolymphoid and epithelial neoplasms - are characterized by recurrent chromosomal translocations. Traditional algorithms in the work-up of these tumors often include a combination of immunohistochemistry panels, cytogenetics, fluorescence in situ hybridization (FISH) and reverse transcriptase-polymerase chain reaction (RT-PCR). Targeted NGS assays are efficient and possess the ability to sequence multiple specimens simultaneously. The ability of a single assay to interrogate hundreds of fusion genes in parallel, including those with known and unknown gene partners, represents a milestone in diagnostic testing. Following implementation of NGS in our department we offer a preliminary view on the diagnostic impact of this assay.

Design: Following successful optimization and validation, we replaced existing RTPCR-based testing with a commercial RNA fusion panel targeting 507 gene associated with diagnostically relevant translocations. RNA was extracted from routine formalinfixed paraffin-embedded tissue is sequenced with 76 base-pair paired-end reads on an Illumina MiSeq at 8 samples per flow cell ( $\sim 3$ million reads per sample). Results were analyzed using both STAR aligner and Manta fusion caller and JAFFA fusion caller utilizing BOWTIE2 aligner.

Results: To date RNA suitable for testing was obtained in $>98 \%$ of cases, with concordance between the histomorphologic differential diagnosis and sequencing results in all cases. In some instances rare fusion breakpoints beyond the range of our existing PCR primers were detected. Additionally, in several cases novel fusion partners were identified. The implementation of this assay allowed more than doubling in the number of cases interrogated by molecular testing over a similar time; however, the turn-around time and cost were greater.

Conclusions: Targeted-NGS represents a powerful adjunct in the diagnosis of bone and soft tissue neoplasms harboring chromosomal translocations. The unprecedented breadth of targets that can be simultaneously interrogated, combined with the benefit of detecting novel fusion partners, makes this a versatile clinical assay that supplants conventional FISH and RT-PCR testing. Although cost and turn-around time are currently greater than prior techniques, it is anticipated these may decrease in the future. 
2152 Correlation of Digital Slides with Raman Spectroscopy : Recommendations for Robust High-Precision Prostate Cancer Detection Vincent Q Trinh, Kelly Aubertin, Andrée-Anne Grosset, Maria C Vladoiu, Catherine St-Pierre, Fred Saad, Mike Jermyn, Frédéric Leblond, Dominique Trudel. Centre Hospitalier de l'Université de Montréal, Montreal, QC, Canada; Centre de recherche $\mathrm{du}$ CHUM \& Montreal Institute of Cancer, Montreal, QC, Canada.

Background: Localization of prostate cancer from a specimen is challenging but necessary to correlate histology to imaging data. Pathologists could rely on whole-mount sections, but this hampers the capacity for automated stains and requires specialized digitalization tools. We describe our correlation technique to test cancer detection algorithms with the non-destructive and label-free Raman spectroscopy (RS).

Design: RS acquisitions are performed on a fresh $4 \mathrm{~mm}$ slice with a hand-held probe and marked by ink. The slice is photographed and re-integrated into a standard workflow in 26x30x5mm cassettes. Scans are obtained with a Leica Aperio imager. Table 1 lists the pre-digital artefacts and digital adjustments before superposing onto the macroscopic image (Figure 1). A machine learning algorithm (MLA) for cancer detection is applied (MATLAB 2016) using support vectors, and cross-validated by the "leave-one-out" method. Prostates are sub-grouped according to the amount of digital corrections needed for correlation, and tested with the same MLA.

Table 1: Pre-digital artefacts, digital adjustments

\begin{tabular}{|c|c|}
\hline Pre-Digital Artefact & Adjustment \\
\hline Tissue warping & $\begin{array}{c}\text { Adjust based on macro-micro correlation: anatomical } \\
\text { landmarks }\end{array}$ \\
\hline $\begin{array}{c}\text { Abnormal transition between } \\
\text { slides }\end{array}$ & Adjust image \\
\hline Positional uncertainty & size of zone \\
\hline Zone borders on edge of slide & Remove if no clean transition \\
\hline No underlying tissue & Remove \\
\hline Major grossing error & \\
\hline Borders on benign and cancer & \\
\cline { 1 - 1 } Suspicion of illogical image & \\
\hline
\end{tabular}

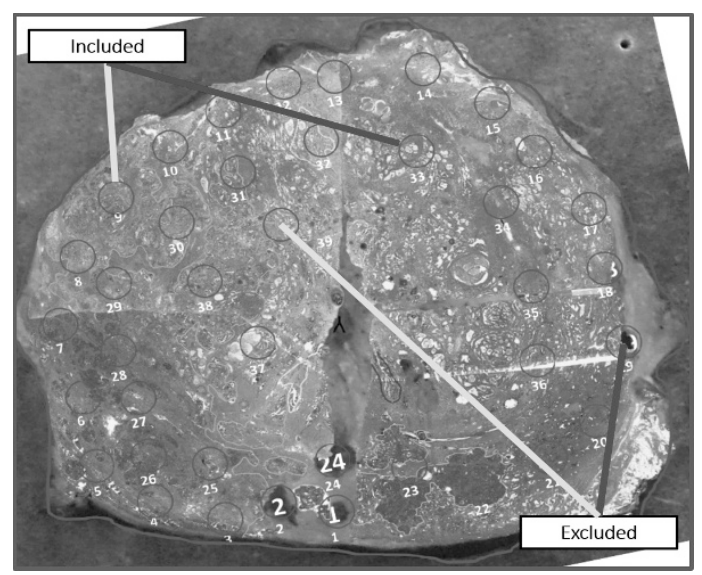

Figure 1: Example of image correlation

Results: RS was acquired for 32 prostates, totaling 1291 acquisitions. Post-digital selection of points identified 937 high-fidelity acquisitions, 786 benign and 151 malignant. The overall MLA performed at $92 \%$ accuracy, $85 \%$ sensitivity and $89 \%$ specificity. When sub-grouped into different imaging classes, their accuracy remained stable at $92 \%$ regardless of the amount of image manipulation.

Conclusions: Precise correlation by the pathologist can lead to robust correlation between gross morphology and histology. Future imaging studies correlating histological data can be inspired by our approach.

2153 Validation and Optimization of an Automated mRNA In Situ Assay Workflow Using RNAscope and Digital Pathology for Gene Expression Analysis of Formalin Fixed Tissues in the Pathology Laboratory

Pamela Villalobos, Barbara Mino, Ignacio I Wistuba, Anna Yemelyanova, Jaime Rodriguez-Canales. The University of Texas M.D. Anderson Cancer Center, Houston, TX.

Background: RNAscope is a Chromogenic in situ hybridization (CISH) method capable of visualizing mRNA gene transcripts and it is compatible formalin-fixed, paraffin-embedded (FFPE) tissue samples. RNAscope technique can be performed on automated stainers (Leica Bond RX) used in the pathology lab. The aim of this study is evaluate an automated workflow using RNAscope for CISH labeling and Aperio Digital Pathology for quantitation of the mRNA transcripts in pathology specimens. Design: Cell lines and whole sections of cervical squamous cell carcinoma were evaluated with PD-L1, HR-HPV, HPV16 and EGFR RNAscope probes by an automated RNAscope protocol using Leica Bond RX autostaining system (Epitope Retrieval 15 $\min 95^{\circ} \mathrm{C}$ and protease incubation $15 \mathrm{~min} 40^{\circ} \mathrm{C}$ ). PPIB and dapB genes were used as positive and negative controls, respectively. For PD-L1, FFPE cell pellets and human tonsil tissue were used as controls for RNAscope (PD-L1 probe) and IHC (clone E1L3N). For HR-HPV, HPV16 and EGFR, vulvar carcinoma cases known to be positive by other methods were included as controls. To validate the staining results, the assay was repeated using manual RNAscope assay and four different automated Leica Bond RX instruments, using identical protocol, reagents and probes. The assay was repeated on a different day to one of the automated instruments to evaluate consistency of the assay (run-to-run variability). For analysis, all RNAscope and IHC slides were scanned (Aperio AT2 scanner) and then analyzed using Image Toolbox software with a novel RNA ISH algorithm (Aperio).

Results: Concordant results were obtained using manual and automated RNAscope assays. The distribution of positive cells with RNAscope was identical on the different tests; however, a difference in intensity of the signal was noticed between the different instruments and from run-to-run on the same instrument. PD-L1 RNA expression pattern matched with the PD-L1 IHC expression in all analyzed cases.

Conclusions: An automated workflow for CISH using RNAscope and digital pathology techniques is a reliable method for detection of various mRNA targets, which has the evident potential to become a powerful tool in the pathology laboratory as an ancillary pathology technique together with classical immunohistochemistry and FISH.

2154 Whole Exome Sequencing of Neuroendocrine Neoplasms of Ovarian Origin

Moiz Vora, Yin Hong, Adam H Greer, Eric X Wei, Rodney Shackelford. Louisiana State University Health Sciences Center, Shreveport, LA.

Background: Neuroendocrine tumors of the ovary consist of a group of heterogeneous malignancies that vary in biologic behavior and prognosis. In recent years a number of new molecular markers and diagnostic tests for the early detection of these tumors have been investigated, but these studies have been based on tumors originating in other anatomic sites. Data on biomarkers of neuroendocrine malignancies of ovarian origin are limited. Here we report on the genomic aberrations of well and poorly differentiated primary ovarian neuroendocrine neoplasms.

Design: Sample tumor DNA was collected from formalin fixed paraffin embedded tissue of four cases after review by H\&E. Two of these cases were well differentiated neuroendocrine tumors and two were non-small cell neuroendocrine carcinomas. DNA libraries were prepared by Illumina's Nextera Rapid Capture Expanded Exome Kit and the pair-end sequencing was performed on Illumina's Nextseq 500 system with Nextseq 500 high output kit ( 150 cycles). Read alignment and variant call analyses were then performed with Illumina's BWA Enrichment, VariantStudio and NextGene software. Results: By BWA Enrichment analysis, we identified 32,519-45,523 single nucleotide variants (snv), 698-1,358 insertion variants, and $830-1,650$ deletion variants. By VariantStudio, these were filtered by passing criteria of read depth (30X), frequency $(5 \%)$, and consequence. Missense variants were 2,847 to 5,961, frameshift truncation were 14 to 41 , and variants to a stop again were 21 to 54 . Comparative assessment by NextGene demonstrated that there were 202 missense or frameshift mutations in 141 genes shared among all four cases. Additional mutations in EGFR, TP53, NTRK1, KMT2D, TSC1, and PTEN were unique to certain cases of well and poorly differentiated neoplasms.

Conclusions: Genomic abnormalities affecting multiple signaling pathways including cell cycle progression, proliferation, and migration, as well as repair of DNA damage are present in primary neuroendocrine neoplasms of the ovary. Most frequently recurrent abnormalities occur in the mucin gene family. Of note a number of the mutations encountered in this study have been reported to play a role in various human malignancies such as medulloblastoma and papillary thyroid carcinoma. The data reported herein may help contribute to the broader effort for a better understanding of the molecular aberrations that drive neuroendocrine carcinogenesis.

2155 A New Pan-Cytokeratin Antibody for Frozen/Fresh Tissues

Jianfu Wang, Yonghua Zhang, Zhiqing Zhang, Angelina Motiee. Novodiax Inc, Hayward, CA.

Background: The pan-cytokeratin (CK) antibody cocktail, clones AE1 and AE3, has been broadly used on FFPE tissues. However, staining of multiple frozen skin and lung tissues with this cocktail consistently gave weak or none staining patterns. Pan-CK antibody has been considered as one of the most important tools for intraoperative evaluations of frozen tissues, such as frozen sentinel lymph node, and therefore its performance on frozen tissues must be reliable. To overcome this problem, a new panCK antibody which works equally well on both FFPE and frozen tissues is needed. Design: A mouse monoclonal antibody clone $\mathrm{C} 100$ was initially identified pan-reactive to multiple cytokeratins on FFTE tissues based upon its IHC staining patterns. The specificity of clone C100 was confirmed by Western Blot using both epithelial and non-epithelial cell lines in comparison with clones AE1/AE3. Clone C100 was then tested on both FFPE and frozen tissues of all major cancer types. Clone $\mathrm{C} 100$ was also directly labeled with a polyHRP and evaluated through a 10-minute IHC protocol.

Results: In western blot analysis, C100 staining pattern overlaps the protein bands of $\mathrm{AE} 1$ and AE3 on lysates of breast and lung cancer cell lines and skin tissues. None of them stained on melanoma cell lysate. Specific and intense IHC stain by clone $\mathrm{C} 100$ was observed on 59 FFPE cancer tissues, including 10 breast, 10 prostate, 9 colon, 10 ovary, 12 uterus, and 8 kidney, and 6 frozen tissues (4 skin tissues and 2 tonsils). Both $\mathrm{C} 100$ and $\mathrm{AE} 1 / \mathrm{AE} 3$ cocktail were labeled with a polyHRP, forming polyHRP-C100 and polyHRP-AE1/AE3. After validating on multiple FFPE tissues, they were tested on 11 frozen tissues with a 10 minute IHC protocol. Satisfactory specific staining was observed with polyHRP-C100 on all frozen tissues tested (skin, tonsil, breast, colon, lung adenocarcinoma, lung squamous cell carcinoma, and uterus tissue). In contrast, polyHRP-AE1/AE3 gave weak to none stain on squamous epithelial cells of frozen skin, tonsils and lung cancer, consistent with our previous observations with unlabeled AE1/AE3 cocktail.

Conclusions: Clone $\mathrm{C} 100$ performed as good as AE1/AE3 on FFPE tissues by IHC and by Western Blot analysis. On frozen tissues, it showed strong and specific immune stain on all squamous and glandular epithelial cells of lung cancer and skin tissues in 
which clones AE1/AE3 showed weak to none stain. It is anticipated that upon thorough tests on more tissues, clone $\mathrm{C} 100$ would be a better tool for assaying intraoperative frozen tissues as well as for cytology tests and study of circulating tumor cells where fresh or lightly fixed cells are used.

2156 Detection of Somatic Mutations in FFPE Tumor Tissues by BaseScope $^{\mathrm{TM}}$ Assay, a Novel Chromogenic RNA In-Situ Hybridization Method

Xiao-Ming M Wang, Xingyong Wu, Liuliu Pan, Jeff Kim, Fan Lin, Nan Su, Emily Park, Robert Monroe, Yuling Luo, Xiao-Jun Ma. Advanced Cell Diagnostics, Newark, CA; Geisinger Clinic, Danville, PA.

Background: The identification of somatic mutations in tumors is important for patien selection for targeted therapies. While high throughput sequencing technologies allow for comprehensive mutation-profiling, they do not permit assessment of intratumoral heterogeneity or the association of genetic alterations with cellular morphology. In addition, DNA mutation status does not predict expression of the mutant allele, which may provide information connecting genotype to phenotype. Therefore, a technology for mutation detection directly in mRNA within the tumor morphological context is desirable. We present a novel chromogenic in situ hybridization (ISH) method for the detection of mutations directly in RNAs in FFPE tissues.

Design: We selected 8 common mutations in the BRAF, KRAS, EGFR, and PIK3CA genes (BRAF V600E; KRAS G12D, G12V and G12A; EGFR L858R and T790M; PIK3CA H1074R and E545K) and designed probes specific for each mutant (mt) and the corresponding wild-type (wt) sequence. We then performed BaseScope, a chromogenic RNA ISH assay, to test the probe specificity in FFPE sections prepared from cell lines with known status for wt and mt alleles. We subsequently tested the assay's ability to detect BRAF V600E mutations in blinded FFPE samples of human colorectal carcinoma (CRC) previously characterized for BRAF status by PCR.

Results: Mutation specific probes generated distinct chromogenic dot signals, representing individual mutant mRNAs in $\mathrm{mt}$ but not wt cell lines. Probes for wt sequences at the same positions resulted in signals in wt and heterozygous mutant cell lines. Blinded analysis of $10 \mathrm{CRC}$ samples ( $5 \mathrm{mt} \& 5 \mathrm{wt}$ ) correctly identified the BRAF V600E status consistent with PCR findings. The spatial distribution of mutant transcript signals is similar across 4 of 5 tumor cases. One tumor showed a decrease in the signals for mutant transcripts in poorly differentiated areas of the tumor.

Conclusions: This novel RNA ISH technology enables detection of somatic mutations and mapping of genetically distinct subpopulations within tumors. It also allows direc visualization of allele-specific expression. This functionality has the potential to further our understanding of tumor heterogeneity and the evolution of resistance mutations. Furthermore, as the mutation status can be visualized chromogenically, this technology has the potential for incorporation into the current anatomic pathology workflow for light microscopic assessment of patients' tumors for specific mutations and possible selection for targeted therapies.

2157 In Situ Classification of Inflammatory Bowel Disease via Mass Spectrometry Tissue Typing

Axel Wellmann, Oliver Klein, Franz Fogt, Tobias Boskamp. Institute of Pathology, Celle, Germany; Berlin-Brandenburger Centrum für Regenerative Therapien, Charité Universitätsmedizin Berlin, Berlin, Germany; University of Pennsylvania and the Presbyterian Medical Center of Philadelphia, Philadelphia, PA; Center for Industrial Mathematics, University of Bremen, Bremen, Germany.

Background: Discrimination of ulcerative colitis (UC) from Crohn's colitis (CC) on histologic features alone can be challenging and often leads to inaccurate initia diagnoses in about $30 \%$ of inflammatory bowel disease (IBD) patients. This is mostly due to an overlap of clinical and histologic features. However, exact diagnosis is not only important for the patient but also has an economic impact. Imaging mass spectrometry (IMS) proteomics promises unique advantages for the analysis of clinical specimens, and these capabilities have been decisive in the advancement of diagnostic medicine and therapy improvement. Therefore, in-situ analysis of IBD tissue sections by IMS were established to identify classifiers for discrimination between "normal",CC, and UC specimens.

Design: Human colon biopsy specimens from patients $(n=28)$ with $\mathrm{CC}$ and UC were collected. Data regarding patient characteristics were used for classification of IBD in the phenotypical subtypes (CC, UC, non-affected). Tissue sections of each phenotype (formalin-fixed and paraffin- embedded tissue) were transferred onto Indium-Tin-Oxide slides. Tryptic-digested material was analyzed by MALDI IMS. IMS data were evaluated by statistical data analysis (probabilistic latent semantic analysis, principal component analysis, receiver operating characteristic (ROC) analysis, linear discrimant analysis) and correlated with the spatial distribution of histologic features. Based on the peptide signatures disease classifiers were identified, which could discriminate between CC, $\mathrm{UC}$ and normal tissue regions.

Results: In this initial study, for the first time, tissue typing via IMS and complex statistical data analysis were applied to determine peptide signature classification models differentiating between "normal" and IBD and within IBD, CC from UC specimens (sensitivity $88 \%$, specificity $73 \%$ ).

Conclusions: Tissue-based proteomic approach using imaging mass spectrometry, combined with patient characteristics provides beneficial and clinically relevant information to improve disease stratification for IBD patients.
2158 Validation of Automated Digital Image Analysis in Evaluating the Prognostic Significance of T-Bet Positive Lymphoid Cells in Lymph Node Negative Breast Cancer

Phillip Williams, Lucine Collins, Dushanthi Pinnaduwage, Irene Andrulis, Anna Marie Mulligan. University of Toronto, Toronto, ON, Canada.

Background: Using tissue microarrays (TMAs) and manual counting, we previously observed the number of intratumoral T-bet positive lymphocytes to be associated with a good prognosis in women with lymph node negative breast cancer. As manual scoring is time-consuming and subject to visual bias we sought to determine whether using an automated digital image analysis algorithm could provide an unbiased, quantitative assessment with equivalent prognostic capabilities.

Design: From a prospectively accrued cohort of women with node negative breast cancer, digitized T-bet stained TMA sections were imported into VisiomorphDP (Visiopharm). A protocol was developed for automated analysis. In brief, first each TMA core had tissue delineated as a region of interest (ROI); second, tissue folds were detected and excluded, automatically; third, T-Bet stained cells were identified automatically by several parameters (color, size and shape) and counted to give an absolute score for each core. Each analyzed TMA core was reviewed by two observers (LC, PW) to ensure: presence of tissue and tumor, tissue quality, and automated analysis quality. Any cases without tissue, tumour, of poor quality or analysis were excluded leaving 225 tumors that were deemed acceptable by automated analysis. Absolute count agreement between the manual and automated methods was assessed by Bland-Altman (B-A) plots with tumors dichotomized into T-bet high and T-bet low. Fisher's exact test or Chi-squared test analysed associations with clinical-pathological variables. Survival analyses were by the Cox proportional hazards model.

Results: B-A plots showed good agreement between the two methods (manual vs automated). T-bet high tumors using both methods were higher grade $(\mathrm{P}=0.0253$, 0.0048 respectively) and significantly associated with hormone receptor negativity ( $E R$, $\mathrm{P}<0.0001$ for both; $\mathrm{PR}, \mathrm{P}=0.0050,0.0121)$, CK5 positivity $(\mathrm{P}<0.0001,0.0139)$, high Ki67 index $(\mathrm{P}=0.0085,0.0054)$ and basal subtype $(\mathrm{P}<0.0001,0.0020)$. At a median follow-up of 102 months, T-bet low tumor status was associated with a reduced diseasefree survival using both methods $(\mathrm{P}=0.0456, \mathrm{P}=0.0468)$. This finding was retained at multivariate analysis $(\mathrm{P}=0.0726,0.0530$ respectively).

Conclusions: Compared with manual counts, automated counting of T-bet positive lymphocytes provides equivalent clinico-pathological associations and prognostic value, thereby representing an unbiased, time efficient and practical method for use in further valuation of this and other immune markers in research and, potentially, clinical settings.

2159 Validation of NGS Detection of the Mutations in the Hematopoietic Disorders with Large Myeloid Panel

Richard Wu, Shuai Wen. Unversity of Miami, Miami, FL.

Background: Hematopoietic disorders harbored different disease associated mutations. About $80 \%$ to $90 \%$ of patients with MDS and $90 \%$ patients with CMML with gene mutation were detected, especially these spliceosomal genes and epigenetic regulators. We have validated the next gene sequence method to detect the mutations in the varieties of hematopoietic disorders with 54 gene panel.

Design: DNAs from twenty three patients were extracted and separated into two portions. Each portion of genomic DNAs was sequenced separately by two different CLIA licensed institutes and the results from two institutes were compared to validate the accuracy of the test. Three samples were run repeatedly with 2 different technologists, 2 different instruments for 5 days to validate the precision of the assay. The detection of lowest allelic frequency was determined by sequencing eight serially diluted samples. Results: Among the total 44 reported variants across those 23 cases, we detected 43 variants with $97.8 \%$ method correlation. For three cases run by two different technologists, at different 5 days and two different instruments, the result concordance was $100 \%$. The lowest allelic frequency that can reliably detected was $5 \%$.

Conclusions: The next gene sequence of 54 gene myeloid panel has been developed and validated. The method can be used in the hematopoietic disorders to detect the disease associated gene mutations with appropriate quality control.

2160 Role of Electronic Microscopy in Evaluation of Hereditary Hyperbilirubinaemia - A Clinicopathological Correlation Study of 24 Cases Japing Zhang, Zichang Yin, Dongfeng Tan. KingMed College of Laboratory Medicine, Guangzhou, Guangdong, China; MD Anderson Cancer Center, Houston, TX.

Background: Hereditary hyperbilirubinaemia is not uncommon group of liver diseases. To make accurate and efficient diagnosis, one needs to combine all relevant information and test results. However, histological diagnosis of hereditary defects of bilirubin metabolism sometimes is not obvious, and clinical presentations as well as laboratory testing of hereditary hyperbilirubinaemia are often unspecific.

Design: This study was designed to systematically evaluate the role of electronic microscopy in assessment and diagnosis of hereditary hyperbilirubinaemia, in conjunction with traditional clinicopathological features. Clinical cases of hereditary hyperbilirubinaemia with recorded ultrastructural studies were enrolled. Clinicopathological featuares and coordination with findings of electronic microscopy were analyzed.

Results: Twenty four consecutive cases with both traditional histology and electronic microscopy were examined. 21 were Gilbert syndrome and 3 Dubin-Johnson syndrome. The male:female ratio was 4.5:1, and onset age ranged 15-54 years old. Major clinical presentations includes jaundice $(100 \%)$, fatigue $(60 \%)$, hepatosplenomegaly $(21 \%)$, and upper quarter dyscomort $(17 \%)$. Lab tests frequently revealed hyperbilirubinaemia $(75 \%)$, but liver functions(ALT, AST, GGT) were usually within normal ranges in most majority of patients(91.7\%), with only slight elevation of ALP in 9 cases(29.2\%). Pathologically, most cases showed unremarkable morphology except pigment 
deposition: Dubin-Johnson pigment was dense and coarse, while among 21 with Gilbert syndrome, only 6 cases demonstrated fine brown pigment granules readily seen perivenular zone. The other 15 cases with equivocal pigment deposition, where electronic microscopy were required to confirm.

Ultrastructrally, hepatocytes of Gilbert syndrome reveal of hypertrophy of the smooth endoplasmic reticulum with finely stippled electron-dense areas and scattered very dense areas; while the Dubin-Johnson pigment is lysosomal, with much bigger very dense areas and smaller finely moderately dense foci than those seen in Gilbert syndrome.

Conclusions: Ultrastructural evaluation of hyperbilirubinaemia plays a role in diagnosis of Gilbert's syndrome. To assess hereditary hyperbilirubinaemia, electronic microscopy is a necessary component along with other relevant clinicopathological features.

2161 The Combined Use of Thermoresponsive NanoVelcro Exosome Purification System and Droplet Digital RT-PCR Enables Highly Sensitive Detection and Dynamic Monitoring of ALK/ROS1 Rearrangement in NSCLC Patients

Yazhen Zhu, Sangjun Lee, Xirun Zheng, Guangiuan Zheng, Hsian-Rong Tseng, Shuang Hou. Guangdong Provincial Hospital of TCM, Guangzhou University of Chinese Medicine, Guangzhou, Guangdong, China; California NanoSystems Institute, Crump Institute for Molecular Imaging, University of California, Los Angeles, Los Angeles, CA.

Background: Liquid biopsy of tumor through isolation of tumor-derived exosomes allows non-invasive, repetitive, and systemic sampling of disease. The key is to establish an efficient and reproducible isolation approach to harvest tumor-derived exosomes from body fluids, paving the way for downstream molecular characterization. The resulting molecular signatures will reveal insight for guiding implementation of targeted therapies. Although significant research endeavors have been devoted to the development of exosome isolation approaches, the existing approaches are of limited clinical impacts due to the limitations such as time-consuming procedures, lack of specificity to distinguish tumor-derived exosomes from those of other tissue origins, and disconnection with proper downstream molecular assays.

Design: In this study, the authors introduced the Thermoresponsive NanoVelcro Assays, capable of isolating tumor-derived exosomes in a highly efficient manner, thus enable a seamless connection with a droplet digital RT-PCR (dd-RT-PCR) platform. The combined use of Thermoresponsive NanoVelcro exosome purification system and dd-RT-PCR enables highly sensitive detection and quantification of ALK/ROS1 rearrangement in non-small cell lung cancer (NSCLC) patients.

Results: The capture efficiency of this unique exosome isolation system was up to $50 \%$ $70 \%$ using NSCLC cell lines with defined ALK and ROS1 rearrangements by modulating capturing markers, flow rates, and the nanowire length of the chips. Subsequently, we were able to isolate cancer exosomes in 5 minutes and successfully detect ALK/ROS1 rearrangement in the isolated exosomes. Exosome isolated from the serum of patients with ALK/ROS1 rearrangement positive NSCLC were shown to contain rearranged ALK/ROS1 transcripts, and we were able to reliably detect and quantify rearranged and wild-type ALK/ROS1 RNA transcripts.

Conclusions: The combined use of Thermoresponsive NanoVelcro exosome purification system and dd-RT-PCR analysis for ALK/ROS1 rearrangement detection and quantification in NSCLC patients represent a valuable new strategy for cancer companion diagnostics, which can be applied to a variety of other gene rearrangements in other cancers.

2162 Quantification of Colonic Mucosal Calretinin Immunostain in Hirschsprung's Disease (HD) by Image Processing and Analysis (IPA) Correlates with Pathologist Visual Interpretation

Chunlai Zuo, Sangtae Ahn, Israel Kasago, Sanaz Ainechi, Suzanne Homan, Christine E Sheehan, Hwajeong Lee. Albany Medical College, Albany, NY.

Background: Calretinin immunostaining of mucosal nerve fibers is used as a surrogate marker of submucosal ganglion cells in the evaluation of HD. Quantification of the calretinin stained mucosal nerve fibers may be useful for studying HD and may aid in objectively identifying the aganglionic zone from the transition zone and normal zone. To evaluate the utility of IPA as a methodology to accurately quantify mucosal nerve fibers, pixel counts of stained areas by IPA were compared with pathologists' visual scoring in the colonic mucosa.

Design: Forty-five images (200X; jpeg format) of calretinin-stained colonic mucosa were taken from $11 \mathrm{HD}$ pull-through specimens. Manual pre-processing preparation was performed using an image editor program. Using MATLAB (MathWorks, Natick, MA), brown stained pixels (RGB $24 ; R>G, R>B$, lightness $<100$, connected size $>50$ pixels) and crypt \& space pixels (k-means clustering in $\mathrm{a}^{*} \mathrm{~b}^{*}$ color space; the cluster with the largest L1 norm of the mean, connected size $>40$ pixels and circles of iteratively increasing size) were segmented. The pixel count was defined as the percentage of the brown stained pixels in all the pixels except for the crypt $\&$ space pixels - pixels of the lamina propria. The original 45 images were independently reviewed by 5 pathologists without knowledge of the pixel count results, and the score 1 (low-), 2 (intermediate-) and 3 (high mucosal calretinin staining) was assigned to each image without defined cutoffs. For each pathologist, the pixel counts of the images with each score were compared by a t-test.

Results: The pixel counts ranged from $0.0116 \%$ to $4.6264 \%$ (mean $0.7696 \%$ ). The range of the mean pixel count was from $0.2328 \%$ to $0.3407 \%, 0.5171 \%$ to $0.9319 \%$, and $1.5782 \%$ to $2.9422 \%$ for score 1,2 and 3 , respectively. The mean pixel count of the images with score 1 was less than that with score 2 and 3 by all $5(100 \%)$ pathologists $(\mathrm{p}<0.01)$. The mean pixel count of the images with score 2 was less than that with score 3 by 4 of $5(80 \%)$ pathologists $(\mathrm{p}<0.05)$.

Conclusions: Pixel count by IPA can be applied to mucosal calretinin staining to quantify nerve fibers. The counts correlate with pathologist visual scoring with statistical significance. In visual scoring, 2-tier scoring (low vs. intermediate \& high) outperformed 3-tier scoring (low vs. intermediate vs. high) with a lower $\mathrm{p}$ value and improved intraobserver variability. The pixel count method by IPA appears useful for studying HD and in providing an objective measure that generates a continuous variable. 